\title{
A KEY TO LARVAE OF SPECIES BELONGING TO THE GENUS DIAMESA FROM ALPS AND APENNINES (ITALY)
}

\author{
BRUNO ROSSARO ${ }^{1, *}$ and VALERIA LENCIONI ${ }^{2}$
}

\footnotetext{
${ }^{1}$ Dipartimento di Scienze per gli Alimenti, la Nutrizione e l’Ambiente, Università degli Studi di Milano, via Celoria, 2, 20133 Milano, Italy

${ }^{2}$ MUSE-Museo delle Scienze, Corso del Lavoro e della Scienza 3, 38123 Trento, Italy

*Corresponding author: bruno.rossaro@unimi.it8
}

\begin{abstract}
A key to species belonging to the genus Diamesa Meigen, 1835 (Diptera, Chironomidae) from the Alps and Apennines (Italy) is presented using characters observable in the fourth-instar larva. The larvae are separated on the basis of qualitative and quantitative characters. At present fifteen species from the Italian Alps are described in all three life stages, but only twelve species groups can be separated as larvae. The separation is based on the length and thickness of anal setae, antennal ratio, head capsule color and few other characters of the labrum and mentum. The shape of mental and mandibular teeth is still a valid taxonomic character, but unfortunately these characters can be rarely used because teeth are often excessively worn in samples collected in the field. Quantitative characters show variability within each species, differing according to the duration of larval development and must be used with caution. The species groups which can be separated in the larval stage are: the dampfi group, which includes $D$. dampfi and $D$. permacra, the latitarsis group including $D$. modesta and $D$. latitarsis, the zernyi group including $D$. zernyi and $D$. vaillanti. The species within each of these groups at present cannot be separated. D. starmachi, D. steinboecki, D. goetghebueri, D. bertrami, D. aberrata, D. incallida, D. cinerella, D. tonsa and $D$. insignipes can be separated from all the other known species in larval stage.
\end{abstract}

Keywords: Chironomidae, Diamesa, dicothomic key, larvae, Italian fauna

\section{Introduction}

The species belonging to the genus Diamesa Meigen, 1835 were described as adult males (Pagast 1947), some also described as females (Willassen and Cranston 1986; Willassen 2005) and as pupal exuviae (Pagast 1947; Langton 1991; Langton and Visser 2003), but few species were described in the larval stage (Schmid 1993; Janecek 1998; Casas and Langton 2001; Lencioni et al. 2007). Unfortunately only larval samples are often collected in hydrobiological studies and the lack of recent keys identifying larvae hinders taking the full advantage of information given by presence or absence of different species in different habitats (Rossaro et al. 2006). Much of the present knowledge about the ecology and distribution of Diamesinae is largely based on the collection of adult and pupal exuviae (Serra-Tosio 1973) or on old even if still valid contributions (Thienemann 1954), so a comprehensive knowledge of the ecological niche of each species is lacking (Lencioni and Rossaro 2005).

The aim of the present paper is to give a description and a key to larvae of the species known in all life stages collected in Italian Alps and Apennines though some material from other European countries (Switzerland, Serbia, Iceland) was also examined. Short notes about the ecology of those described species is given here, while more detailed autoecological notes will be matter of future contributions.

\section{Material and Methods}

Larvae were fixed in $70 \%$ ethylalcohol or in $4 \%$ formalin, transferred in acetic acid and in a mixture of phenol : xylol $3: 1$ (Wirth and Marston 1968), mounted in a drop of Canada balsam on a microscope slide and dissected, separating the head from the body; the body was mounted in lateral or dorso-ventral position under a separate cover slip; the posterior part of the body including the 8th9th segments and posterior parapods was often mounted separately in dorsal position with gentle pressure, to better examine the anal setae. The head was mounted under a separate cover slip, after squashing, so that the labral setae could be more clearly seen. Sometime it was necessary to dissect the labrum with clypeal sclerite and frons from the rest of the head and mount it in dorsal position.

Measurements were made using a LEICA DM LS B2 optic microscope at different magnification $(\times 40-\times 1000)$ connected to a LEICA DFC320 camera. The specimens measured were previously mounted on slides that are archived in the Department of Food, Environmental and Nutritional Sciences of Università degli Studi di Milano (Italy) (about 1000 slides) and in the MUSE-Museo delle Scienze of Trento (Italy) (about 2000 slides). Measurements, if not otherwise specified, are given in microns $(\mu \mathrm{m})$ : mean \pm standard deviation, minimum, maximum values and number of observations are reported. The measurements refer to the specimens mounted on slides that are a small 
sample with respect to all the specimens collected, thus the given ranges are probably smaller than the true ones.

The information about material collected is given as the name of river, stream or lake followed by the values of latitude North and longitude East, given in Italian Gauss Boaga coordinate system, calculated as the mean over all the sampled stations belonging to the same catchment, the station name, the sampling date, the stage collected $(\mathrm{L}=$ larva, $\mathrm{Pe}=$ pupal exuvia, $\mathrm{P}=$ pupa, Prep $=$ prepupa, $\hat{\sigma}=$ male, $q=$ female) and the number of specimens collected are given thereafter. Data were filed in a relational database in Microsoft Access.

\section{Diamesa (Meigen 1835)}

\section{Diagnosis}

The characters described were examined observing larvae in the fourth-instar.

Body length 5-12 $\mathrm{mm}$. Coloration of the body from yellow, yellow green, brown to black in living larvae, body color is not preserved in mounted material. Head capsule yellow in some species, with a large black occipital margin, tips of mandibles and mentum black; more or less extended brown spots and extended dark areas at the base of mentum may be present; head capsule entirely brown-black in other species, with light areas around eyes; head capsule color is maintained in mounted material. Head setae simple.

Body setae weak, very long (about $150 \mu \mathrm{m}$ ) in D. dampfi, D. permacra, moderately long (about $70 \mu \mathrm{m}$ ) in $D$. aberrata.

\section{Antenna}

With 5 segments, third segment generally annulated, blade a little shorter than flagellum, reaching either the half or the distal margin of segment 4 ; in some species as long as flagellum, sometime longer than flagellum, accessory blade about $3 / 4$ of blade length, reaching either the base or the half of segment 3 , sometime reaching the base of segment 4, apex of second antennal segment bearing a style, reaching either the base or the half or the distal margin of segment 4 , and 2 small peg sensilla (Lauterborn organs). First antennal segment with a ring organ near the base of antenna, beside the ring organ two antennal setae are present, a proximal one near the ring organ and a distal one near the apex of the first segment. The distances of ring organ and antennal setae from the base of first antennal segment are given in species descriptions. $\mathrm{AR}$ variable between less than one to about two.

\section{Labrum}

SI triangular with a large basis. SII and SIII thin, SII sometime with a large scale at its base, SIII simple, bifid with two simple or divided branches, SIVa present and developed, SIVb small, labral lamellae present, consisting of scales with simple, bifid or branched apices, often arranged in three groups, groups of branched chaetae on each side, chaeta media present lateral to labral lamellae, lateral to chaeta media about five spinules are present on each side, apically divided into 2 or more points. Pecten epipharyngis consisting of 5 helmet shaped scales, with 2-3 pairs of chaetulae laterales. Ungula U-shaped with a well developed basal sclerite.

Premandible with 5-7 apical teeth, lateral spine present and branched.

\section{Mentum}

Median tooth single or double, if simple sometime with a small median indentation, from 7 to 12 lateral teeth. The most lateral tooth may be very small. Anterior margin convex, straight or concave. Ventromentum with lateral expansions (ventromental plates), setae submenti arising anteriorly with respect to caudal extremity of ventromentum (in species descriptions this is expressed with negative values of the distance between caudal margin of ventromentum and setae submenti), in D. aberrata, D. incallida and in D. starmachi (Schmid 1993) setae submenti are posterior to caudal extremity of ventromentum at a short distance of about 5-10 $\mu \mathrm{m}$, in D. dampfi group setae submenti are well posterior with respect to caudal margin of ventromentum, at a distance of about $30 \mu \mathrm{m}$. Both distance of seta submenti from ventromental plates and from caudal margin of ventromentum are given in species descriptions, the former can be measured with more accuracy, because in some specimens the posterior extremity of ventromentum is not well defined.

Mentum teeth often worn, so difficult to use in species identification.

\section{Premento-hypopharyngeal complex}

Labial palp with 5 sensilla; ligula deeply divided into branches, median prementum simple and triangular, paraligula formed by a group of setae, paraligula and ligula appear as 3 brushes, lateral chaetulae of prementum present, pecten hypopharyngis consisting of several transverse rows of apically serrated plumose and pointed scales (Sæther and Andersen 2013).

\section{Mandible}

With an apical and 4 median teeth, in some species the first two more developed than the 3rd and 4th.

Ventral seta subdentalis simple, seta interna with 25-30 simple branches, inserted medially on the dorsal surface of mandible. Mandibular teeth often worn, so difficult to use in species identification.

\section{Maxilla}

Palp as long as broad, with 10-11 sensillae; chaetulae of palpiger and lamellae of galea setose with 5-6 sensillae and lamellae; pecten galearis absent; lacinia short with overlapping long chaetulae; appendix setae absent; simple setae maxillaris (antaxial setae) present (Sæther and Andersen 2013). 


\section{Parapods}

Anterior and posterior parapods with apical claws, yellow in anterior, black in posterior parapods. Posterior parapods with a small seta in the apical third.

\section{Procercus}

Very reduced or absent, 1-4 anal setae and 1 lateral setae arising from the procercus area, only in $D$. dampfi group procercus is not reduced and bears 6 apical setae and 1 lateral seta.

\section{Species descriptions}

The information about each species is given only for characters that differ in each species, characters common to all species are given in the genus diagnosis.

\section{Diamesa permacra (Walker 1856)}

(Fig. 1)

Body length: $9110 \pm 0.49(9110-9111, \mathrm{n}=12)$, with very long setae $>150 \mu \mathrm{m}$, head capsule and throat uniformly yellow or brownish, only occipital margin, tip of mentum and mandible black, head length: $610 \pm 3.00$ (610-611, $\mathrm{n}=12)$, head width: $339 \pm 0.00(339-339, \mathrm{n}=1)$.

Antenna: AR: $1.80 \pm 0.12(1.59-2.10, \mathrm{n}=12)$, blade length: $26.2 \pm 2.04(23.8-30.4, \mathrm{n}=12)$, accessory blade: $20.4 \pm 2.58(17.3-23.7, \mathrm{n}=12)$, distance of ring organ from the base of the 1 st antennal segment: $10.0 \pm 1.06$ $(9.01-11.7, \mathrm{n}=12)$, proximal antennal seta: $11.9 \pm 0.86$ $(11.4-13.3, \mathrm{n}=12)$, distal antennal seta: $49.2 \pm 5.18$ $(41-52.5, \mathrm{n}=12)$, style: $10.2 \pm 1.39(8-12.2, \mathrm{n}=12)$, style thickness: $1.95 \pm 0.37(1.3-2.73, \mathrm{n}=12)$, 1st antennal segment: $63.1 \pm 3.45(58.2-71.7, \mathrm{n}=12)$, thickness of 1 st antennal segment: $19.4 \pm 1.81(17.6-22.5, \mathrm{n}=12)$, combined length of segments 2-5: $35.2 \pm 2.70$ (30.9-40.3, $\mathrm{n}=12)$, length of antennal segments 2-3-4-5: $18.3 \pm 0$ $(18.3-18.3, \mathrm{n}=1), 8.93 \pm 0(8.93-8.93, \mathrm{n}=1), 6.35 \pm 0$ $(6.35-6.35, \mathrm{n}=1), 5.82 \pm 0(5.82-5.82, \mathrm{n}=1)$.

Labrum with simple setae SIII.

Mentum: length: $294 \pm 26.9$ (251-317, $\mathrm{n}=12)$, ventromentum not reaching setae submenti, distance from ventromental plates to setae submenti: $55.9 \pm 2.22$ (55.9$55.9, \mathrm{n}=12)$, distance between caudal margin of ventromentum and setae submenti: $31.3 \pm 0(31.3-31.3, \mathrm{n}=1)$, median mental tooth almost fused with the 1st lateral, sometime with a notch, 9 lateral teeth if the median tooth is not fused, if the median tooth is fused with the first laterals only 7-8 lateral teeth are visible, the last tooth is very small.

Posterior parapods length: $516 \pm 0(516-516, \mathrm{n}=1)$, procercus present and developed, with 6 anal setae and 1 lateral seta, procercus length: $26.6 \pm 8.80(7.46-32.1, \mathrm{n}=$ $12)$, procercus diameter: $30.5 \pm 3.37(25.1-35.3, \mathrm{n}=12)$, anal setae length: $502 \pm 58.9(375-544, \mathrm{n}=12)$, anal setae thickness: $3.58 \pm 0.34(3.11-4.31, \mathrm{n}=12)$.
Material examined: upper Oglio river, 5117231N, 1602875E, Oglio alta valle Camonica, 15/5/2000, L 1; 1/7/2000, Pe 1; Orta lake, Acqualba, Lagna stream, 50 77673N, 1450825E, Acqualba04, 3/2/1977, L 1; Acqualba02, 15/1/1976, L 7; Acqualba02, 24/1/1976, L 1; Acqualba03, 24/1/1976, L 14; Acqualba02, 15/2/1976, L 1; Acqualba01, 28/2/1976, L 7; Acqualba02, 28/2/1976, Љ઼ P 1; Acqualba03, 28/2/1976, L 17; Acqualba02, 2/3/1976, q Pe 2; Acqualba01, 27/3/1976, L 2; Acqualba03, 27/3/1976, L 27; Acqualba01, 1/4/1976, L 3; Acqualba02, 0/7/1976, L 1; Acqualba02, 27/7/1976, L 3; Agogna, Grua stream, 5066562N, 1450737E, Grua01, 25/3/1981, 1; Grua02, 1/1/1978, L 3; Grua03, 1/1/1978, L 1; Grua03, 15/1/1978, L 1; Grua03, 1/3/1977, L 2; Grua04, 1/3/1977, L 5; Grua04, 15/3/1977, L 1; Grua04, 15/4/1977, L 1; Grua04, 0/5/1977, L 1; Grua03, 1/5/1977, L 4; Grua04, 0/6/1977, L 1; Grua04, 1/6/1977, L 1; Grua02, 0/12/1977, L 1; Grua04, 0/12/1977, L 1; Grua02, 1/12/1977, L 2; Grua04, 1/12/1977, L 2; Gallavesa stream, 5073888N, 1537551E, Gallavesa, 12/11/1977, L 1; Brembo river, 5081387N, 1550439E, Brembo03S G Bianco, 4/5/2000, L 1; Serina, 12/4/1982, L 1; Brembo01Carona, 12/4/1982, L 1; Brembo03S G Bianco, 16/3/1981, $q$ 1; Brembo02Lenna, 16/3/1981, L 1; Lambro river, 5083077N, 1523273E, Lamb02Barni, 27/12/1977, L 1; Lamb02Barni, 10/3/1976, L 2; Agogna stream, 5038645N, 1473957E, Agogna 04NO, 0/12/1980, L 1; Aterno river river, 4700535N, 1873330E, Raiale, 3/4/1994, L 2.
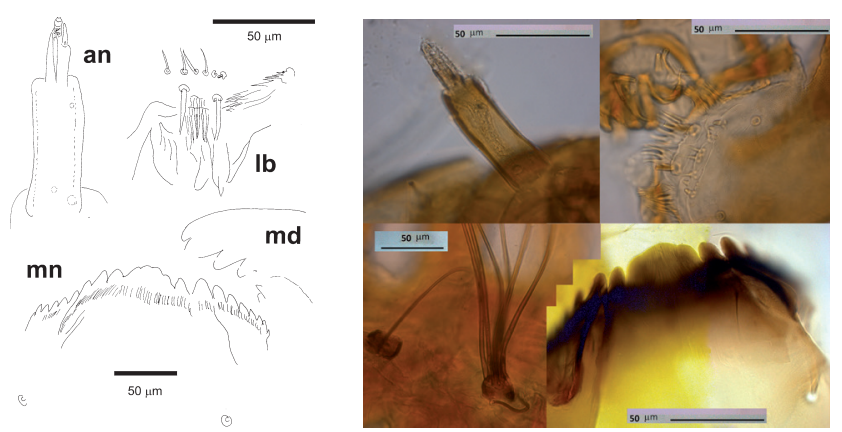

Fig. 1 Left: Diamesa permacra, an: antenna - lb: labrum - mn: mentum md: mandible; right: Diamesa permacra, antenna, labrum, anal setae, mentum.

\section{Diamesa dampfi (Kieffer 1924)}

(Fig. 2)

Body length: $6405 \pm 3.11(6400-6406, \mathrm{n}=8)$, with very long setae about $150 \mu \mathrm{m}$, head capsule and throat uniformly yellow or brownish, only occipital margin, tip of mentum and mandible black, head length: $563 \pm 0.17$ (563-564, $\mathrm{n}=8)$, head width: $313 \pm 0.09(312-314, \mathrm{n}=8)$.

Antenna: AR: $1.97 \pm 0.13(1.73-2.07, \mathrm{n}=8)$, blade length: $28.9 \pm 1.63(26.7-30.4, \mathrm{n}=8)$, accessory blade about $3 / 4$ antennal blade: $22.4 \pm 1.41(18.9-23, \mathrm{n}=8)$, distance of ring organ from the base of the 1st ant. segm.: $11.8 \pm 2.23(9.33-13.8, \mathrm{n}=8)$, proximal antennal seta: $14.3 \pm 4.16(10.4-18.2, \mathrm{n}=8)$, distal antennal seta: 56.9 
$\pm 5.26(52-61.8, \mathrm{n}=8)$, style: $11.9 \pm 0.28(11.6-12.3$, $\mathrm{n}=8)$, style thickness: $2.08 \pm 0.56(1-2.8, \mathrm{n}=8)$, 1 st antennal segment: $69.8 \pm 4.06(63.3-73.3, \mathrm{n}=8)$, thickness of 1st antennal segment: $21.8 \pm 2.11(18.7-25.1, \mathrm{n}=8)$, combined length of segments $2-5: 35.3 \pm 1.07$ (33.0-36.5, $\mathrm{n}=8)$, length of antennal segments $2-3-4-5: 18.3 \pm 0$ $(18.3-18.3, \mathrm{n}=8), 8.93 \pm 0(8.93-8.93, \mathrm{n}=8), 6.35 \pm 9.49$ $(6.35-6.35, \mathrm{n}=8), 5.82 \pm 9.49(5.82-5.82, \mathrm{n}=8)$.

Labrum with simple setae SIII.

Mentum: length: $288 \pm 35.5(243-327, \mathrm{n}=8)$, with a median tooth often fused with the 1st lateral, appearing as a large median tooth, more than 3 times wide $(40 \mu \mathrm{m})$ with respect to the most adiacent lateral tooth $(13 \mu \mathrm{m})$ when fused, sometime well divided into 3 teeth, the median one three times large $(18.5 \mu \mathrm{m})$ the first lateral one $(6.8 \mu \mathrm{m})$, often a bit notched, 9 lateral mental teeth if the median tooth is fused with the 1st lateral, 10 when median tooth is separated, the 10th tooth is very small and may be absent. Ventromental plates not much expanded laterally, ventromentum not reaching setae submenti, distance from ventromental plates to setae submenti: $65 \pm 0$ (65-65, $\mathrm{n}=8$ ), distance between caudal margin of ventromentum and setae submenti: $34.6 \pm 2.11(32.0-36.9, \mathrm{n}=8)$.

Posterior parapods length: $516 \pm 0(516-516, \mathrm{n}=1)$, procercus present and developed, with 6 anal setae and 1 lateral seta, procercus length: $20.2 \pm 0.62(19.1-21.5, \mathrm{n}$ $=8$ ), procercus diameter: $33.4 \pm 3.63(26.4-35.3, \mathrm{n}=8)$, anal setae length: $520 \pm 46.7(423-544, \mathrm{n}=8)$, anal setae thickness: $4.25 \pm 0.80(2.66-5.54, \mathrm{n}=8)$.

Material examined: upper Adda river Mallero stream SO, 5127789N, 1561613E, Adda 38 Mallerolc, 6/2/1999, L 1; Adda 38 Mallero1c, 14/5/1999, L 1; Adda 38 Mallero3, 14/5/1999, L 3; Adda 38 Mallerola, 3/7/1999, L 11; Adda 38 Mallero3, 3/7/1999, L 20; Adda 38 Mallerola, 30/7/1998, L 1; Adda 38 Mallerola, 25/8/1998, L 3; Adda 38 Mallero3, 25/8/1998, L 27; Adda 38 Mallero3, 25/9/1998, L 92; Adda 38 Mallero2, 17/10/1998, L 1; Adda 38 Mallero3, 17/10/1998, L 31; Adda 38 Mallero3, 6/11/1998, L 29; Adda 38 Mallero1b, 12/12/1998, L 1; Adda 38 Mallero3, 12/12/1998, L 38; Adda 38 Mallero, 26/6/1983, L 2; upper Adda river Masino stream SO, 5125435N, 1552669E, Adda 46 Mello 01, 25/2/1979, L 8; upper Adda river Caronno, 5103812N, 1570876E, CaseraA, 6/11/1998, L 1; CaseraC, 6/11/1998, L 1; upper Oglio river, $5126744 \mathrm{~N}, 1616843 \mathrm{E}, \mathrm{S}$ Apoll ruscello, 4/8/2009, L 5; Frigidolfo, 15/12/2008, L 10; S Apoll ruscello, 27/12/2007, L 17; Frigidolfo, 2/1/1981, L 3; Vezza st 03, 16/2/1981, L 1; Vezza st 02, 16/2/1981, L 1; Bighera, 10/8/1981, ô P 1; Bighera, 20/8/1981, L 30; Frigidolfo, 6/4/1980, L 10; Frigidolfo, 7/4/1980, L 1; Frigidolfo, 15/4/1980, Pe 1; S Apoll upper spring, 19/7/1980, Pe 45; Vione, 20/7/1980, L 1; S Apoll upper spring, 26/7/1980, §̋ P 5; S Apoll upper spring, 4/3/1979, Pe 10; S Apoll ruscello, 13/4/1979, L 1; S Apoll upper spring, 19/5/1979, Pe 30; Vezza st 02, 30/12/1979, L 1; S Apoll upper spring, 14/11/1978, L 10; Avisio stream, 5128882N, 1630927E, Vermigliana, 3/5/2014, L 1; Vermigliana, 28/12/2007, prep 1; Vermigliana, 0/11/1979, prep 1; Vermigliana, 14/4/1978, L 1; Care Alto glacier, Conca stream (car), $5108398 \mathrm{~N}, 1625954 \mathrm{E}$, car 2C7 J, 21/6/2005, L 1; car 2C7 L, 19/9/2005, L 1; Dora Veny glacial stream, 5070309N, 1330795E, Col De La Seigne, 9/9/1998, L 50; Mt Blanc, 8/9/1996, ô 3; Miage, 7/7/1995, L 1; Livigno, 5148697N, 1592835E, Adda 30 Foscagno, 10/9/2007, L 50; Parco Naz. Gran Paradiso (PNGP) Orco river, 5036950N, 1377323E, F2-TRUC-1, 16/8/2010, L 66; Marmore glacial stream, 5087560N, 1393950E, Cervinia, 16/9/1995, đ̂ 1; Po river PC, 4996980N, 1611433E, Po river Piacenza, 26/6/1983, L 1; Po river Piacenza, 23/4/1981, L 1.
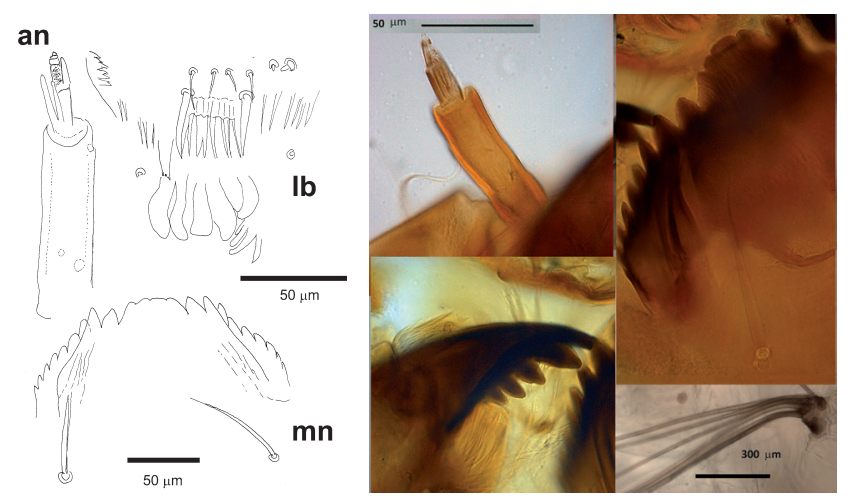

Fig. 2 Left: Diamesa dampfi, an: antenna - lb: labrum - mn: mentum; right: Diamesa dampfi, antenna, mentum, mandible, anal setae.

\section{Diamesa steinboecki (Goetghebuer 1933)}

(Fig. 3)

Body length: $5091 \pm 273(4772-5302, \mathrm{n}=10)$, but large larvae living more than one year can be larger, until $8 \mathrm{~mm}$, head entirely black, head length: $658 \pm 159$ $(534-843, \mathrm{n}=10)$, head wide: $365 \pm 88.4(297-468, \mathrm{n}=10)$.

Antenna: short, antennal blade almost reaching apex of antenna or a little shorter, accessory blade reaching the base of segment 4th, style longer than segment 3, reaching half of segment 4, AR: $1.46 \pm 0.28(1.18-2.07, \mathrm{n}=10)$, blade: $18.7 \pm 2.43(14.1-20.3, \mathrm{n}=10)$, accessory blade: $13.0 \pm 3.41(6.68-15.6, n=10)$, distance of ring organ from the base of the 1st ant. segm.: $4.25 \pm 2.52$ (2.29-7.7, $\mathrm{n}=10)$, proximal antennal seta: $4.60 \pm 0.20(4.41-4.8, \mathrm{n}$ $=10)$, distal antennal seta: $16.9 \pm 1.08(14.5-18, \mathrm{n}=10)$, style: $8.46 \pm 0.38(7.94-9, \mathrm{n}=10)$, style thickness: 3.08 $\pm 0.01(3.05-3.09, \mathrm{n}=10), 1$ st antennal segment: $36.9 \pm$ $5.35(30.2-43.3, \mathrm{n}=10)$, thickness of 1 st antennal segment: $12.8 \pm 0.32(12-13, \mathrm{n}=10)$, combined and separated lengths of segments 2-5: $26.2 \pm 7.13(17.4-36.0, \mathrm{n}$ $=10)$, length of antennal segments $2-3-4-5: 8.91 \pm 1.87$ $(8.91-8.91, \mathrm{n}=10), 3.15 \pm 4.68(3.15-3.15, \mathrm{n}=10), 2.53$ $\pm 4.68(2.53-2.53, \mathrm{n}=10), 2.87 \pm 4.68(2.87-2.87, \mathrm{n}=10)$.

Labrum with multibranched setae SIII, SII with a large scale at its base, labral lamellae toothed at apex, chaeta media short, semicircular.

Mandible: with the apical and the first two inner teeth very long, equal in length, or the 2 nd is the longest, $3 \mathrm{rd}$ and 4th inner teeth shorter, length of inner teeth: 2nd > 1 st $>3$ rd $>4$ th. 
Mentum: length: $206 \pm 14.2(190-221, \mathrm{n}=10)$, with a paired median tooth and 10 (11) lateral teeth, median teeth often worn and mentum appears with a large median tooth and a smaller number of lateral teeth, distance from ventromental plates to setae submenti: $33.6 \pm 0.44$ (33-34.3, $\mathrm{n}=10)$, setae submenti arising anteriorly with respect to caudal extremity of ventromental plates, so the distance between caudal margin of ventromentum and setae submenti is less than $0:-24 \pm 0(\mathrm{n}=1)$.

Posterior parapods length: $445 \pm 5.99$ (445-445, $\mathrm{n}=$ $10)$, procercus absent, anal setae very short, thick, spinelike, about $6(5-8) \%$ long with respect to mentum length, anal setae length: $13.5 \pm 7.29(3-25, \mathrm{n}=10)$, anal setae thickness: $3.37 \pm 0.39(3-3.74, \mathrm{n}=10), 0-1,1-2,2-2,3-1$ setae on the right and on the left side respectively, with a lateral seta at most $30.0-33.5 \mu \mathrm{m}$ long.

Material examined: upper Adda river Mallero stream SO, 5128571N, 1615524E, Adda 31 Sforzell3, 16/7/2003, Pe 5; Vedretta Carè Alto (car), 5108398N, 1625954E, car 1C3 I, 19/9/2005, L 3; Parco Naz. Gran Paradiso (PNGP) Orco river, 5036950N, 1377323E, NEROEUGIO, 30/6/2009, L 22; Sarca Genova river TN, 5117326N, 1623091E, Bedole Rif, 13/9/1990, Pe 1.
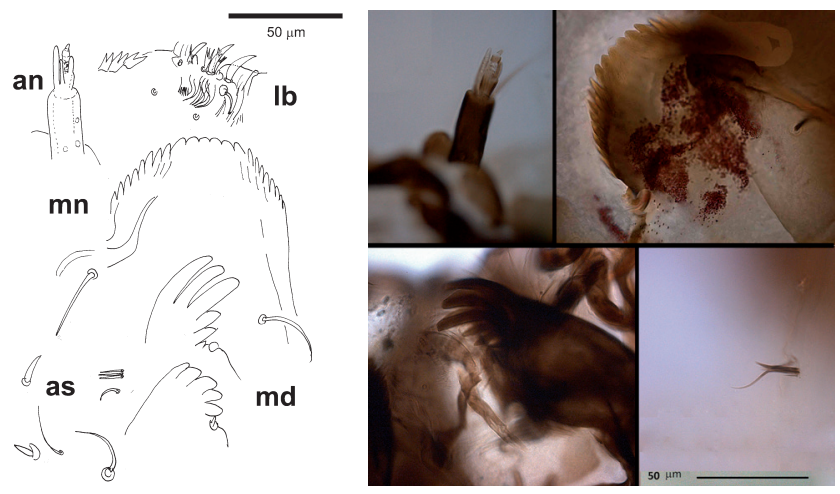

Fig. 3 Left: Diamesa steinboecki, an: antenna - lb: labrum - mn: mentum - md: mandible - as: anal setae; right: Diamesa steinboecki, antenna, mentum, mandible, anal setae.

\section{Diamesa goetghebueri (Pagast 1947)}

(Fig. 4)

Body length: $7553 \pm 694(4772-7717, \mathrm{n}=18)$, but larvae living more than one year can be much larger, head capsule entirely black, head length: $843 \pm 0.38$ (843-844, $\mathrm{n}=18)$, head width: $468 \pm 0.21(468-468, \mathrm{n}=18)$.

Antenna: short, AR: $1.30 \pm 0.29(0.84-2, \mathrm{n}=18)$, blade length: $24.1 \pm 3.56(18.6-32, \mathrm{n}=18)$, accessory blade: $14.1 \pm 2.38(10-18, n=18)$, distance of ring organ from the base of the 1st ant. segm.: $6.59 \pm 1.64$ (2.29-9, $\mathrm{n}=18)$, proximal antennal seta: $6.21 \pm 2.33(4.41-9.1$, $\mathrm{n}=18)$, distal antennal seta: $19.0 \pm 1.47(16.6-21, \mathrm{n}=18)$, style: $8.70 \pm 1.25(6-11, \mathrm{n}=18)$, style thickness: $2.65 \pm$ $0.01(2.6-2.66, \mathrm{n}=18), 1$ st antennal segment: $38.9 \pm 11.0$ (22.1-55.7, $\mathrm{n}=18)$, thickness of 1st antennal segment: $22.8 \pm 0.38(22-23, \mathrm{n}=18)$, combined length of segments $2-5: 29.7 \pm 3.87(23.6-37, n=18)$, length of antennal seg- ments $2-3-4-5: 12.7 \pm 3.22(9.14-15.7, \mathrm{n}=18), 4.67 \pm$ $0.15(4.09-4.73, \mathrm{n}=18), 2.66 \pm 0.42(2.26-3.87, \mathrm{n}=18)$, $2.20 \pm 0.51(1.62-3.07, \mathrm{n}=18)$.

Labrum with multibranched setae SIII, SII with a large scale at its base.

Mandible: with 1st lateral tooth more developed than others, the other lateral teeth decreasing in size, 1st $>2 \mathrm{nd}$ $>3$ rd $>4$ th.

Mentum: length: $227 \pm 33.0(193-304, \mathrm{n}=18)$, with a paired median tooth and 10 lateral teeth, median tooth often worn out, sometime appears as a single median tooth, 1st lateral tooth a little longer than the median and the 2nd lateral one, 2nd lateral may be shorter than the $3 \mathrm{rd}$, distance from ventromental plates to setae submenti: $41.0 \pm 3.05(35.1-46.2, \mathrm{n}=18)$, setae submenti arising anteriorly with respect to caudal extremity of ventromental plates, so the distance between the caudal margin of ventromentum and setae submenti is less than $0:-8.1 \pm 3.77(-15--6.1, \mathrm{n}=1)$.

Posterior parapods length: $755 \pm 318(445-1064, n=18)$, Anal setae 4-3 on the right side, 3-3 on the left side, thin, short, very much reduced with a longer lateral seta, procercus absent, anal setae length: $20.1 \pm 8.45$ (7.93-41.2, $\mathrm{n}=18)$, anal setae thickness: $1.32 \pm 1.18(0.5-3.45, \mathrm{n}=18)$.

Material examined: Suisse Rhone, $5158070 \mathrm{~N}$, 1453786E, RH3B Gletsch 26/9-3/10/1999; upper Oglio river, 5121534N, 1605373E, Vezza st 02, 21/3/2014, L 1; Vezza st 01 monte, 23/8/1980, Pe 2; Dora Baltea river, 5080029N, 1406854E, Lys, 29/6/1987, Ô P 10; Dora Veny glacial stream, 5068650N, 1331469E, Estellette, 10/9/1995, ô 1; Sarca river, 5107511N, 1622276E, SanGiuliano, 10/8/1996, L 44; Sarca Genova river TN, 5169807N, 1793511E, Pinzolo, 31/7/2002, ô 2; Lobbia vedretta, 12/9/1990, ठ̂ 1; Bedole Rif, 14/8/1979, ठ̊ 1.

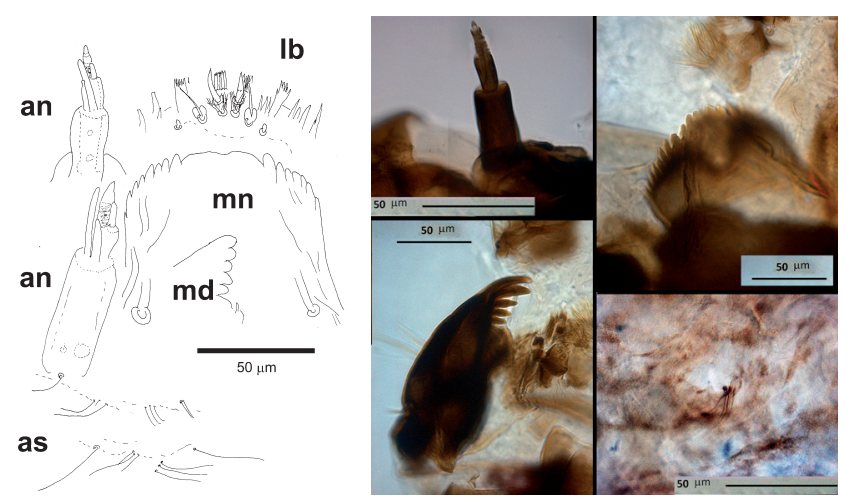

Fig. 4 Left: Diamesa goetghebueri, antenna, mentum, mandible, anal setae; right: Diamesa goetghebueri, an: antenna - lb: labrum - mn: mentum - md: mandible - as: anal setae.

\section{Diamesa latitarsis (Goetghebuer 1921), Diamesa modesta (Serra-Tosio 1968)}

(Fig. 5)

Body length: $10491 \pm 1625$ (7000-11500, $n=13)$, head capsule entirely black, head length: $660 \pm 0.35$ (660-661, $\mathrm{n}=13)$, head width: $367 \pm 0.19(366-367, \mathrm{n}=13)$. 
Antenna: short, AR: $1.23 \pm 0.14(1.13-1.68, \mathrm{n}=13)$, blade length: $25.0 \pm 2.05(23.1-29.1, \mathrm{n}=13)$, accessory blade: $14.7 \pm 1.11(13.1-16.6, \mathrm{n}=13)$, distance of ring organ from the base of the 1st antennal segment: 6.84 $\pm 0.55(5-7, \mathrm{n}=13)$, proximal antennal seta: $7.43 \pm$ $0.95(6.9-9.1, \mathrm{n}=13)$, distal antennal seta: $22.1 \pm 2.60$ $(19.7-27, \mathrm{n}=13)$, style: $7.80 \pm 1.03(7-9.83, \mathrm{n}=13)$, style thickness: $2.37 \pm 0.12(2.27-2.66, \mathrm{n}=13), 1$ st antennal segment short: $36.5 \pm 4.23(31.6-48, \mathrm{n}=13)$, thickness of 1 st antennal segment: $12.9 \pm 0.27(12-13, \mathrm{n}=13)$, combined length of segments 2-5: $29.5 \pm 1.63$ (26.8-32.3, n $=13)$, length of antennal segments $2-3-4-5: 15.8 \pm 1.84$ $(15.8-15.8, \mathrm{n}=13), 7.68 \pm 2.77(7.68-7.68, \mathrm{n}=13), 3.76 \pm$ $1.38(3.76-3.76, \mathrm{n}=13), 3.85 \pm 4.62(3.85-3.85, \mathrm{n}=13)$.

Labrum with multibranched setae SIII, SII with a large scale at its base.

Mandible: first inner tooth more developed than others, 1 st $>2$ nd $>3$ rd $>4$ th.

Mentum: length: $219 \pm 15.7(209-253, \mathrm{n}=13)$, with a paired median tooth and 11 lateral teeth, with a convex anterior margin, distance from ventromental plates to setae submenti: $39.4 \pm 1.16(36.8-39.9, \mathrm{n}=13)$, setae submenti arising anteriorly with respect to caudal extremity of ventromental plates, so the distance between caudal margin of ventromentum and setae submenti is less than $0:-6.5 \pm 1.60(-7.1--2.9, \mathrm{n}=3)$.

Posterior parapods length: $592 \pm 141$ (553-1064, $\mathrm{n}=13$ ), procercus absent, anal setae length: $43.3 \pm 9.9$ $(27.8-59.1, \mathrm{n}=13)$, anal setae thickness: $\varnothing: 1.50 \pm 0.19$ $(1-1.65, \mathrm{n}=13)$.

Material examined: Avio lake, 5117319N, 1608019E, Avio, 9/8/1978, L 11; Mallero stream, 5125830N, 1549840E, CampoMoro, 8/8/1997, L 11; upper Adda river Caronno, 5103812N, 1570876E, CaseraC, 2/7/1999, L 4; CaseraC, 14/8/1998, L 13; CaseraC, 10/10/1998, L 1; upper Oglio river, 5121188N, 1616949E, Pisgana glacier, 2/1/1979, L 15; Frigidolfo, 30/7/1978, o P 1; Benedetto, 3/8/1978, L 1; Pisgana glacier, 3/8/1978, L 1; S Apoll spring, 24/9/1978, ô P 1; Care Alto glacier, Conca stream (car), 5108398N, 1625954E, car 1C3 H, 9/8/2005, L 1; car 1C8 E, 1/8/1997, Pe 1; Vedretta Cornisello, 5110178N, 1622312E, vdr 4V3 D, 1/6/1997, L 1; vdr 4V4 D, 1/6/1997, L 1; Dora Ferret glacial stream, $5081210 \mathrm{~N}, 1347345 \mathrm{E}$, ruscbeta65, 4/7/1995, L 10; Dora Veny glacial stream, 5070432N, 1334111E, Combal alplake, 21/7/1998, L 4; Col De La Seigne, 22/7/1998, L 15; Combal alplake, 14/9/1997, L 1; Combal alplake, 6/9/1996, L 2; Combal alplake, 26/3/1995, L 1; Combal alplake, 3/7/1995, L 1; Brouillard, 6/7/1995, Pe 1; Combal alplake, 7/7/1995, L 10; Mt Blanc, 7/7/1995, L 2; Combal alplake, 26/9/1985, L 5; Parco Naz. Gran Paradiso (PNGP) Orco river, 5036451N, 1359847E, F2GAV-1, 18/7/2010, L 6; F2-GAV-2A, 18/7/2010, L 66; F2-RHEM-1, 26/7/2010, L 33; F2-CARP6-1, 25/8/2010, L 80; F2-CARP6-2B, 25/8/2010, L 3; F2-CAR-2C, 25/8/2010, L 66; F2-ROC-2A, 18/9/2010, L 29; F2LEV-1, 18/9/2010, L 33; F2-NOA-1, 29/9/2010, L 6; Sarca
Genova river TN, 5117744N, 1629879E, Nardis cascata, 13/9/1990, Pe 11; Bedole Rif, 12/9/1980, ô 1; Venerocolo, 5115584N, 1614483E, Venerocolo 01 alplake, 25/9/1978, L 11; Elvo, 5044666N, 1419337E, Elvo Sordevolo, 21/4/2001, Pe 4; Elvo Sordevolo, 6/5/2001, L 1; Cherio river, Endine lake, 5070494N, 1572555E, EndineG01qt, 0/8/1979, L 11; EndineG02ql, 0/8/1979, L 11; EndineG01qt, 9/8/1979, L 11; Brembo river, 5081387N, 1550439E, Brembo03S G Bianco, 17/4/2000, ô 1; Sangro river, 4633561N, 1904694E, PNA04, 19/5/1978, L 3; Sangro river, 4645145N, 1912368E, PNA11 Profluo, spring, 21/5/1978, L 1; Vomano river, Mavone stream 4716144N, 1883507E, Isola del Gran Sasso, 20/3/1993, ô 1; Isola del G sasso, 4/12/1992, L 1.
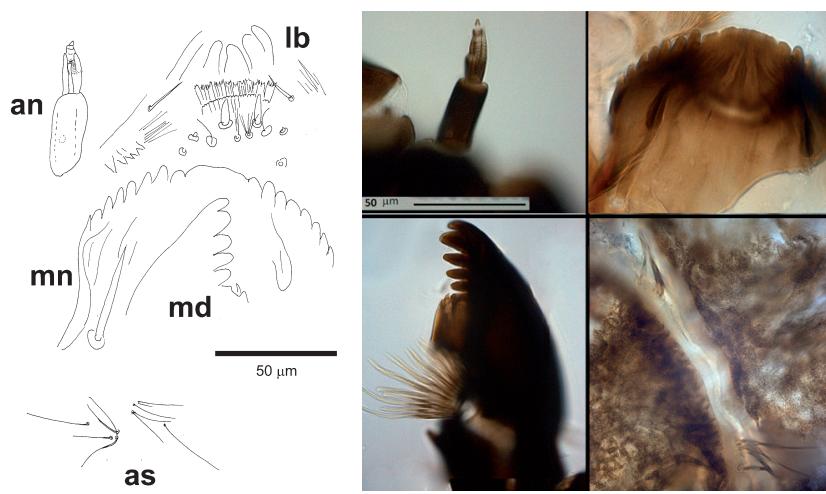

Fig. 5 Left: Diamesa latitarsis, antenna, mentum, mandible, anal setae; right: Diamesa latitarsis, an: antenna - lb: labrum - mn: mentum - md: mandible - as: anal setae.

\section{Diamesa bertrami (Edwards 1935)}

(Fig. 6)

Body length: $6833 \pm 234(6700-7232, \mathrm{n}=28)$, head capsule entirely black, head length: $403 \pm 79.6$ (166-486, $\mathrm{n}=28)$; head width; $224 \pm 44.2(92.2-270, \mathrm{n}=28)$.

Antenna: longer than in D. latitarsis, but shorter than in D. aberrata, AR: $1.55 \pm 0.34(0.86-2.06, \mathrm{n}=28)$; blade length: $24.1 \pm 1.52(19.0-26, \mathrm{n}=28)$; accessory blade: $14.7 \pm 1.95(11-19, \mathrm{n}=28)$; distance of ring organ from the base of the 1 st antennal segment: $6.67 \pm 4.06$ (3.512.7, $\mathrm{n}=28)$; proximal antennal seta: $7.2 \pm 3.09(5.2-12$, $\mathrm{n}=28)$; distal antennal seta: $25.3 \pm 1.98(22-27, \mathrm{n}=28)$; style: $9.10 \pm 0.66(8-11.4, \mathrm{n}=28)$; style thickness: $1.98 \pm$ 0.29 (1.41-2.3, $\mathrm{n}=28)$; 1st antennal segment: $46.2 \pm 9.94$ (23.1-63.3, $\mathrm{n}=28)$; thickness of 1st antennal segment: $11.2 \pm 0.22(11-12, \mathrm{n}=28)$; combined length of segments: $2-5 ; 30.2 \pm 5.30(24.1-46.7, \mathrm{n}=28) ; 13.2 \pm 0.74$ $(11.8-13.6, \mathrm{n}=28) ; 34.8 \pm 14.7(7.17-42.4, \mathrm{n}=28) ; 5.04$ $\pm 0.30(4.46-5.2, \mathrm{n}=28) ; 3.47 \pm 0.43(2.66-3.7, \mathrm{n}=28)$.

Labrum with multibranched setae SIII, SII without a large scale at its base.

Mandible with lateral teeth very long, decreasing in size in the sequence $2 \mathrm{nd}>1$ st $>3 \mathrm{rd}>4$ th.

Mentum: length $221 \pm 32.3(153-257, \mathrm{n}=28)$, with a concave median margin, a paired median tooth [single in some specimens], a 2nd lateral tooth longer than 
the 1 st and 10 lateral teeth, distance from ventromental plates to setae submenti; $35.9 \pm 1.44(35.9-35.9, \mathrm{n}=28)$, setae submenti arising anteriorly with respect to caudal extremity of ventromental plates, so the distance between caudal margin of ventromentum and setae submenti is submenti is lessless than $0:-20 \pm 7.23(-18--22, \mathrm{n}=1)$.

Posterior parapods length: $829 \pm 65.3(713-874, \mathrm{n}=$ 28); procercus absent; anal setae spiniform, anal setae length: $78.3 \pm 20.2(18.9-133, \mathrm{n}=28)$; anal setae thickness: $2.43 \pm 0.83(1.2-4, \mathrm{n}=28)$.

Material examined: upper Ticino river, 5147220N, 1468824E, Lavizzara, 5/7/2007, L 2; upper Adda river Mallero stream SO, 5141560N, 1616559E, Adda 31 Forni, 25/9/2013, L4 3; Adda 31 Forni, 18/9/2001, Pe 10; Adda 38 Mallerola, 25/9/1998, L 1; Adda 38 Mallero, 9/8/1997, 仓 1; Adda 38 Bignami, 12/8/1997, L 1; Adda 38 Mallero, 12/8/1997, ô 1; Adda 01 Viola, 0/7/1995, L 1; Adda 01 Viola, 17/7/1985, L 1; Adda 38 Mallero, 22/6/1983, L 2; Adda 31 Forni, 24/8/1980, + Pe 1; Braulio Stelvio, 11/8/1977, ô P 1; Braulio Stelvio, 14/8/1977, o 1; Mallero stream, 5125830N, 1549840E, CampoMoro, 9/8/1997, ô 1; Avisio stream, 5131154N, 1711791E, Travignolo, 5/8/1988, Pe 1; Travignolo, 6/8/1988, L 20; upper Oglio river, $5121116 \mathrm{~N}, 1613094 \mathrm{E}$, Vione, 0/7/1985, L 5; Vione, 0/10/1985, L 1; Vione, 0/12/1985, L 6; Vezza st 02, 3/1/1981, L 1; Frigidolfo, 15/2/1981, prep 1; S Apoll upper spring, 28/8/1981, L 2; Vezza st 02, 15/4/1980, L 1; Vezza st 02, 4/8/1980, L 1; Vezza st 03, 23/8/1980, L 2; Vezza st 02, 23/8/1980, L 1; Vezza st 02, 0/12/1980, L 1; S Apoll ruscello, 2/1/1979, prep 1; S Apoll ruscello, 19/5/1979, Pe 5; Bighera, 8/9/1979, L 1; Vezza st 02, 8/9/1979, L 1; Aviolo alplake, 8/9/1979, L 1; Vezza st 03, 9/9/1979, L 1; Vezza st 03, 6/11/1979, Pe 13; S Apoll ruscello, 7/11/1979, L 1; Vezza st 02, 22/4/1978, L 1; Frigidolfo, 30/4/1978, L 2; S Apoll ruscello, 5/6/1978, L 1; S Apoll ruscello, 10/6/1978, L 3; S Apoll ruscello, 3/7/1978, Pe 4; Vezza st 02, 29/7/1978, L 2; S Apoll ruscello, 30/7/1978, Pe 13; S Apoll spring, 30/7/1978, đ̂ P 1; Vezza st 02, 1/8/1978, ㅇ 1; Pisgana glacier, 1/8/1978, L 2; Bighera, 3/8/1978, L 1; Bighera, 12/8/1978, L 1; Benedetto, 12/8/1978, L 2; Vezza st 02, 14/8/1978, PeM 1; Vezza st 02, 16/8/1978, L 31; Vezza st 02, 18/8/1978, + Pe 1; Vezza st 02, 24/8/1978, L 21; S Apoll ruscello, 24/9/1978, L 9; S Apoll spring, 14/11/1978, ㅇ 1; Avisio stream, 5128882N, 1630927E, Vermigliana, 2/1/1995, L 1; Vermigliana, 4/3/1979, L 2; Vermigliana, 14/4/1979, ㅇ 1; Vermigliana, 27/12/1979, L 1; Vermigliana, 23/4/1978, L 6; Vermigliana, 28/12/1978, L 9; Care Alto glacier, Conca stream (car), 5108398N, 1625954E, car 1C2 D, 1/6/1997, Pe 10; car 1C3 D, 1/6/1997, Pe 2; car 2C6 D, 1/6/1997, L 3; car 2C7 D, 1/6/1997, L 5; car 1C0 D, 1/6/1997, L 1; car 1C2 E, 1/8/1997, Pe 12; car $1 \mathrm{C} 3 \mathrm{E}, 1 / 8 / 1997$, Pe 4 ; car 1C5 E, $1 / 8 / 1997$, Pe 1 ; car 2C6 E, 1/8/1997, L 59; car 2C7 E, 1/8/1997, L 3; car 1C0 E, 1/8/1997, Pe 68; car 1C2 9 , 1/9/1997, Pe 47; car 1C3 $9,1 / 9 / 1997$, Pe 6; car 1C5 + , 1/9/1997, Pe 1; car $1 \mathrm{C} 8$ \%, 1/9/1997, Pe 1; car 2C6 + , 1/9/1997, L 3; car $2 \mathrm{C} 7$ 9, 1/9/1997, L 1; car 1C0 , 1/9/1997, Pe 50; car
1C3 A, 1/6/1996, L 2; car 1C4 A, 1/6/1996, L 1; car 1C5 A, 1/6/1996, L 2; car 1C8 A, 1/6/1996, L 2; car 2C6 A, 1/6/1996, L 1; car 2C7 A, 1/6/1996, L 2; car 1C2 B, 1/8/1996, L 25; car 1C3 B, 1/8/1996, L 1; car 1C4 B, 1/8/1996, L 1; car 1C5 B, 1/8/1996, Pe 5; car 1C8 B, 1/8/1996, Pe 1; car 2C6 B, 1/8/1996, L 6; car 2C7 B, 1/8/1996, L 9; car 1C1 B, 1/8/1996, L 12; car 1C2 C, 1/9/1996, Pe 20; car 1C4 C, $1 / 9 / 1996$, Pe 2 ; car 1 C5 C, 1/9/1996, Pe 1; car 1 C8 C, 1/9/1996, Pe 2; car 2C6 C, 1/9/1996, L 7; Vedretta Cornisello, 5110178N, 1622312E, vdr 4V1 G, 1/6/1998, L 1; vdr 4V2 G, 1/6/1998, L 4; vdr 4V3 G, 1/6/1998, L 14; vdr 4V1 H, 1/8/1998, L 8; vdr 4V2 H, 1/8/1998, L 20; vdr 4V3 H, 1/8/1998, L 126; vdr 4V4 H, 1/8/1998, L 2; vdr 4V1 I, 1/9/1998, L 2; vdr 4V2 I, 1/9/1998, L 1; vdr 4V3 I, 1/9/1998, L 5; vdr 4V4 I, 1/9/1998, L 2; vdr 4V2 D, 1/6/1997, L 4; vdr 4V0 E, 1/8/1997, L 23; vdr 4V1 E, 1/8/1997, L 1; vdr 4V2 E, 1/8/1997, L 1; vdr 4V3 E, 1/8/1997, L 5; vdr 4V1 ㅇ, 1/9/1997, L 1; vdr 4V2 , 1/9/1997, L 1; vdr 4V3 의, 1/9/1997, L 15; vdr 4V4 9, 1/9/1997, L 8; Dora Baltea river, 5080029N, 1406854E, Lys, 30/6/1987, L 4; Dora Ferret glacial stream, 5081210N, 1347345E, Ferret briomadic43, 7/7/1995, L 10; Dora Veny glacial stream, 5070309N, 1330795E, Col De La Seigne, 21/7/1998, L 65; Estellette, 21/7/1998, L 13; La Le Blanche, 21/7/1998, L 3; Estellette, 22/7/1998, L 3; Col De La Seigne, 8/9/1998, L 1; Estellette, 8/9/1998, L 2; La Le Blanche, 8/9/1998, L 1; Estellette, 9/9/1998, L 261; Col De La Seigne, 12/9/1997, L 1; Estellette, 12/9/1997, L 89; Col De La Seigne, 13/9/1997, L 1; Estellette, 13/9/1997, L 87; La Le Blanche, 13/9/1997, L 88; Col De La Seigne, 14/9/1997, L 1; Estellette, 14/9/1997, ô 1; Col De La Seigne, 6/9/1996, L 48; Estellette, 6/9/1996, L 160; La Le Blanche, 7/9/1996, L 15; Estellette, 8/9/1996, \& Pe 1; Estellette, 11/9/1995, L 2; La Le Blanche, 11/9/1995, \& 1; Alpine lake Ghiacciato, 5107511N, 1622276E, Alplake Ghiacciato in, 10/8/1996, L 3; Alplake Ghiacciato in, 21/8/1996, L 3; Lys glacial stream, 5092954N, 1409631E, Gressoney, 30/6/1987, ô 1; St Jacques, 21/9/1980, L 1; Niscli glacial stream, $5110178 \mathrm{~N}, 1622312 \mathrm{E}$, niscl 3N0 E, 1/8/1997, L 11; niscl 3N1 E, 1/8/1997, L 36; niscl 3N2 E, 1/8/1997, L 10; niscl 3N0 , 1/9/1997, L 2; niscl 3N1 ㅇ, 1/9/1997, L 2; Sesia river, 5080029N, 1406854E, Rosa Bors, 27/7/1979, L 1; Olen stream, 15/9/1979, $q$ 1; Marteltal, Plima stream, 5151291N, 1630445E, MartelTal, 16/9/1995, PeF 1; Avisio stream, 5149717N, 1712427E, Canazei, 6/8/1988, L 1; Sarca Genova river TN, 5169807N, 1793511E, Pinzolo, 31/7/2004, đิ 1; Bedole Rif, 11/9/1990, Љ 1; Bedole Rif, 12/9/1990, Pe 1; Marmore glacial stream, 5087560N, 1393950E, Cervinia, 20/9/1980, L 1; Agogna, Grua stream, 5066562N, 1450737E, Grua01, 0/3/1987, L 2; Grua03, 15/3/1977, L 1; Cherio river, Endine lake, 5070494N, 1572555E, EndineG03qt, 25/2/1981, L 11; EndineG01ql, 28/4/1981, L 33; EndineG02qt, 17/6/1981, L 11; EndineG03ql, 17/6/1981, L 44; EndineG03qt, 17/6/1981, L 11; EndineG03ql, 28/8/1981, L 11; EndineG01ql, 2/7/1980, L 11; EndineG01qt, 12/8/1980, L 11; EndineG03qt, 12/8/1980, L 33; EndineG03ql, 18/9/1980, 
L 11; EndineG03qt, 15/10/1980, L 44; EndineG02ql, 9/8/1979, L 11; Gallavesa stream, 5073888N, 1537551E, Gallavesa, 8/12/1979, L 1; Lambro river, 5071949N, 1518133E, Lamb06Caslino, 26/11/2004, L 1; Lamb06Caslino, 17/12/2004, P 1; Lamb04Asso, 26/1/1978, L 1; Lamb06Caslino, 3/2/1977, L 1; Lamb04Asso, 3/2/1977, L 1; Lamb06Caslino, 16/11/1977, o 1; Lamb05SCalocero, 16/11/1977, ㅇ Pe 1; Lamb03Lasnigo, 16/11/1977, L 2; Agogna stream, 5038676N, 1467446E, Agogna 93, 8/7/1996, ㅅ 1; Po river PC, 4996980N, 1611433E, Po river Piacenza, 1/8/1981, L 1; MassaCarrara, 4888177N, 1635997E, stream near Abetone pass, 2/3/1981, L 1; Sagittario stream, 4652152N, 1905025E, PNA08 Tasso stream, 20/5/1978, L 11.
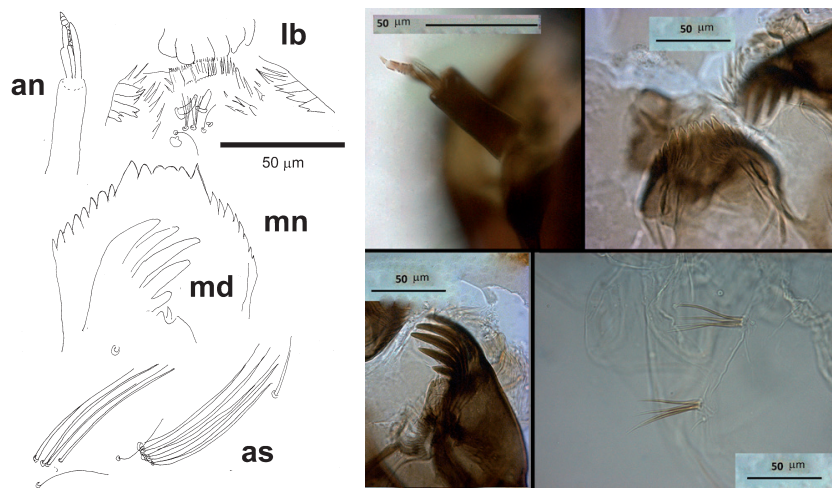

Fig. 6 Left: Diamesa bertrami, antenna, mentum, mandible, anal setae; right: Diamesa bertrami, an: antenna - lb: labrum - mn: mentum - md: mandible - as: anal setae.

\section{Diamesa aberrata (Lundbeck 1898)}

(Fig. 7)

Body length $5494 \pm 624(4900-7405, \mathrm{n}=18)$, abdominal segments with long setae $>70 \mathrm{~mm}$, head capsule entirely black, head length: $449 \pm 199(206-604, \mathrm{n}=18)$, head width: $249 \pm 110(114-335, \mathrm{n}=18)$.

Antenna: longer than in D. latitarsis and D. bertrami, AR high, often higher then 2, $2.10 \pm 0.43(1.37-2.73, \mathrm{n}=$ 18), blade length: $26.7 \pm 1.72(25.3-32, \mathrm{n}=18)$, accessory blade: $18.8 \pm 0.82(18.4-22, \mathrm{n}=18)$, distance of ring organ from the base of the 1st antennal segment.: $12 \pm 2.91$ $(11-20, \mathrm{n}=18)$, proximal antennal seta: $14.9 \pm 2.74(14-22.5$, $\mathrm{n}=18)$, distal antennal seta: $43.9 \pm 2.65(43-51.2, \mathrm{n}=$ 18), style: $9.49 \pm 1.22(8.88-13, \mathrm{n}=18)$, style thickness: $2.19 \pm 0.01(2.18-2.22, \mathrm{n}=18), 1$ st antennal segment segm about $80 \mu \mathrm{m}$ long, $73.5 \pm 20.8(26.5-98.2, \mathrm{n}=18)$, thickness of 1 st antennal segment: $11.0 \pm 1.35(10-16, \mathrm{n}=$ 18 ), combined length of segments $2-5: 34.3 \pm 4.65$ (19.2$40.3, \mathrm{n}=18)$, length of antennal segments $2-3-4-5: 16.2$ $\pm 2.12(13.6-17.9, \mathrm{n}=18), 25.3 \pm 13.9(14.5-42.4, \mathrm{n}=18)$, $5.2 \pm 9.13(5.2-5.2, \mathrm{n}=18), 3.7 \pm 9.13(3.7-3.7, \mathrm{n}=18)$.

Labrum with bifid setae SIII, 6-8 labral lamellae consisting of a high number of scales with simple apices, with 4-5 lateral spines.

Mentum: length: $229 \pm 47.1(153-328, \mathrm{n}=18)$, with 11-12 lateral, the 4-5 innermost lateral teeth arranged in a straight line, median tooth paired, distance from ventromental plates to setae submenti: $45.4 \pm 1.46$ (44-46, $\mathrm{n}=18$ ), setae submenti arising posteriorly with respect to caudal extremity of ventromental plates, but at a short distance $[11.9 \pm 2.20(9.2-14.4, \mathrm{n}=18)]$ from caudal margin of ventromentum.

Mandible with lateral teeth decreasing in size in the sequence $3 \mathrm{dr}>2 \mathrm{nd}>1$ st $>4$ th, [26:25:24:18 $\mu \mathrm{m}]$, but often worn out.

Posterior parapods length: $749 \pm 2.33$ (749-749, $\mathrm{n}=18$ ), about $730 \pm 10 \mu \mathrm{m}$ long, procercus length: 3.06 $\pm 0(3.06-3.06, \mathrm{n}=18)$, procercus diameter: $14.6 \pm 0$ (14.6-14.6, $\mathrm{n}=1$ ), anal setae length: $91.4 \pm 17$ $(63.1-140, \mathrm{n}=18)$, anal setae thickness: $3.85 \pm 1.32$ $(2.19-5.34, \mathrm{n}=18)$.

Material examined: Toce river, 5097995N, 1457436E, Grande Verbania, 21/12/1993, L 1; upper Adda river SO, 5128571N, 1615524E, Adda 31 Sforzell1, 17/9/2003, L 10; Adda 31 Sforzell1, 18/9/2001, ô Pe 3; Adda $38 \mathrm{Mal}$ lero2, 30/7/1998, L 4; Adda 38 Mallero3, 30/7/1998, L 3; Adda 38 Mallero2, 25/8/1998, L 1; Adda 38 Mallero2, 25/9/1998, L 1; Adda 31 Forni, 28/8/1975, L 1; upper Oglio river, $5121534 \mathrm{~N}, 1605373 \mathrm{E}$, Vezza st $02,17 / 12 / 2008$, L 1; DezzoE3, 0/6/1989, L 8; S Apoll ruscello, 12/7/1980, ð઼ P 1; S Apoll upper spring, 12/7/1980, त̂ Pe 1; S Apoll spring, 12/7/1980, L 1; Pisgana glacier, 18/8/1980, Pe 1; S Apoll spring, 4/3/1979, L 1; Bighera, 10/8/1979, L 1; Grande valle 02 BS, 14/8/1979, L 10; Ercavallo stream, 16/8/1979, Pe 83; S Apoll spring, 24/8/1979, L 1; Vezza st 02, 9/9/1979, L 2; Vezza st 02, 6/11/1979, Pe 22; S Apoll spring, 7/11/1979, đ̊ Pe 11; Temu', 9/6/1978, Pe 1; Temu', 10/6/1978, Pe 1; S Apoll spring, 10/6/1978, L 3; Care Alto glacier, Conca stream (car), 5108398N, 1625954E, car 1C3 G, 21/6/2005, L 7; car 1C0 B, 1/8/1996, L 1; Dora Veny glacial stream, 5070309N, 1330795E, Col De La Seigne, 7/9/1996, L 1; Estellette, 7/9/1996, L 1; Col De La Seigne, 8/9/1996, L 2; Mt Blanc, 6/7/1995, đ 1; La Le Blanche, 7/7/1995, L1 3; Estellette, 8/7/1995, L 1; Brenva springfredda, 8/7/1995, L 3; Estellette, 8/9/1995, L 1; Negro lake Gavia pass, 5134079N, 1612854E, Gavia psso lago Negro, 24/8/1979, L 11.111; Parco Naz. Gran Paradiso (PNGP) Savaranche glacial stream, 5043870N, 1359449E, Savaranche Rif Vitt Eman alpha, 20/9/1980, Pe 1; Presena, 5121263N, 1620805E, Presena, 13/9/1990, L 11.111; Sarca Genova river TN, 5117326N, 1623091E, Bedole Rif, 14/8/1978, L 2; Cuc Pian, 14/8/1978, L 1; Orta lake, Acqualba, Lagna stream, $5066533 \mathrm{~N}, 1454626 \mathrm{E}, \mathrm{La}-$ gna, 3/4/1979, L 1; Brembo river, 5077237N, 1558208E, Ola Alben Oltre il Colle, 16/11/1982, L 1; 0N, 0E, Serina, 11/4/1981, L 1; Lambro river, 5073804N, 1519423E, Lamb05SCalocero, 26/1/1978, L 2; Lamb05SCalocero, 3/2/1977, L 17; Lamb04Asso, 16/11/1977, ㅇ 1; Lamb01Magreglio, 11/3/1976, L 67; Lamb02Barni, 11/6/1976, L 1; Lamb01Magreglio, 22/11/1976, L 1; Bormida river, 4896408N, 1429434E, Calizzano Mereta A2, 12/3/1990, L 4; Osiglia, 12/3/1990, L 1; Calizzano Caragna A3, 
1/5/1990, L 1; Calizzano Mereta A2, 20/3/1989, L 3; Osiglia, 20/3/1989, L 29; Millesimo Acqua Fredda C1, 20/3/1989, L 1; Calizzano Caragna A3, 20/3/1989, L 1; Osiglia, 15/5/1989, L 1; Po river PC, 4996980N, 1611433E, Po river Piacenza, 25/6/1983, L 1; PoTO, 4985692N, 1316089E, Piano del Re, 25/6/1983, L 1; Nera river, $4715717 \mathrm{~N}, 1803886 \mathrm{E}$, Marmore cascata, 4/12/1992, L 1; Aso stream, 4768169N, 1853339E, Aso01, 18/4/1981, prep 1; Aso02, 18/4/1981, L 1; Aso02, 17/4/1980, L 1; Aso04, 17/4/1980, 1; Aso01, 29/1/1979, ㅅ P 1; Aso02, 29/1/1979, L 2; Aso01, 28/3/1979, 1; Aso02, 28/3/1979, Pe 1; Sangro stream, 4636951N, 1898941E, PNA02, 20/5/1978, L 4; PNA04, 20/5/1978, ô Pe 5; Sagittario stream, 4645145N, 1912368E, PNA11 Tasso mte spring, 20/5/1978, Pe 1; PNA10 Tasso spring, 20/5/1978, o 1; PNA09 Tasso, 20/5/1978, ㅇ Pe 1 .

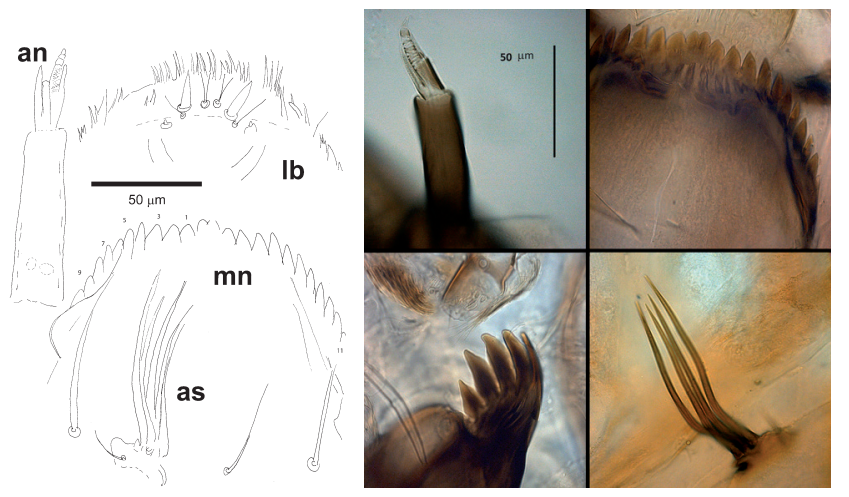

Fig. 7 Left: Diamesa aberrata, antenna, mentum, mandible, anal setae; right: Diamesa aberrata, an: antenna - lb: labrum - mn: mentum - as: anal setae.

\section{Diamesa incallida (Walker 1856)}

(Fig. 8)

Body length: $7951 \pm 749$ (7405-8826, $\mathrm{n}=5)$, head capsule light brown, head length: $671 \pm 99.6$ (604-826, $\mathrm{n}=5)$, head width: $373 \pm 55.3(335-459, \mathrm{n}=5)$.

Antenna: long, with the 3 rd segment characterized by 2 large rings, AR very high: $2.27 \pm 0.31(1.98-2.78$, $\mathrm{n}=5)$, blade length: $24.2 \pm 3.66(20.3-27.8, \mathrm{n}=5)$, accessory blade: $19.2 \pm 0.50(18.7-19.8, \mathrm{n}=5)$, distance of ring organ from the base of the 1st antennal segment: $12.8 \pm 3.95(10-17.2, \mathrm{n}=5)$, proximal antennal seta: 13.2 $\pm 5.15(10.9-22.5, \mathrm{n}=5)$, distal antennal seta: $50.0 \pm 12.3$ $(41-63.5, \mathrm{n}=5)$, style: $7.97 \pm 2.10(5.47-11.3, \mathrm{n}=5)$, style thickness: $2.3 \pm 0.05(2.25-2.35, \mathrm{n}=5), 1$ st antennal segment: $79.6 \pm 7.53(67.7-86.7, \mathrm{n}=5)$, thickness of $1 \mathrm{st}$ antennal segment: $17.9 \pm 0.15(17.6-18, \mathrm{n}=5)$, combined length of segments $2-5: 35.2 \pm 2.66(31.0-37.4, \mathrm{n}=5)$, length of antennal segments 2-3-4-5: $19.8 \pm 0$ (19.8$19.8, \mathrm{n}=5), 14.5 \pm 0(14.5-14.5, \mathrm{n}=5), 5.2 \pm 0(5.2-5.2$, $\mathrm{n}=5), 3.7 \pm 0(3.7-3.7, \mathrm{n}=5)$.

Labrum with bifid setae SIII, SII without a large scale at its base, chaeta media well developed.

Mentum: length: $302 \pm 52.8(245-358, \mathrm{n}=5)$, with the 4 th median teeth forming a plate well separated from the other lateral teeth, distance from ventromental plates to setae submenti: $49 \pm 0(49-49, \mathrm{n}=5)$, setae submenti arising posteriorly with respect to caudal extremity of ventromental plates, but at a short distance [5 $\pm 1(4-6, n$ =5)] from caudal margin of ventromentum.

Posterior parapods length: $552 \pm 0(552-552, \mathrm{n}=5)$, procercus length: $8.98 \pm 0(8.98-8.98, \mathrm{n}=5)$, procercus diameter: $20.6 \pm 0(20.6-20.6, \mathrm{n}=5)$, anal setae length: $276 \pm 17.3(253-294, \mathrm{n}=5)$, anal setae thickness: $7.44 \pm$ $0.63(6.30-7.72, \mathrm{n}=5)$.

Material examined: upper Oglio river, 5128571N, 1615524E, S Apoll spring, 4/7/2009, L 20; S Apoll spring, 27/12/2007, L 3; Vezza st 02, 21/4/1981, L 1; S Apoll spring, 28/8/1981, L 1; S Apoll spring, 24/2/1980, L 40; S Apoll spring, 18/7/1980, L 30; S Apoll spring, 18/2/1979, L 30; S Apoll ruscello, 4/3/1979, Pe 2; S Apoll spring, 13/4/1979, L 30; S Apoll spring, 19/5/1979, L 30; Vezza st 02, 10/8/1979, L 29; Aviolo alplake, 19/8/1979, § P 1; Cherio river, Endine lake, 5070494N, 1572555E, EndineG01qt, 19/8/1979, ô P 11; EndineG02ql, 19/8/1979, L 11; EndineG01qt, 8/9/1979, đ P 11; EndineG02ql, 8/9/1979, L 11; Lambro river, 5083077N, 1523273E, Lamb02Barni, 16/11/1977, L 2; Nure, 4942433N, 1539660E, Nure, 29/2/1980, L 1; Taro river, 4936509N, 1573000E, Taro 3, 4/5/2000, L 1.

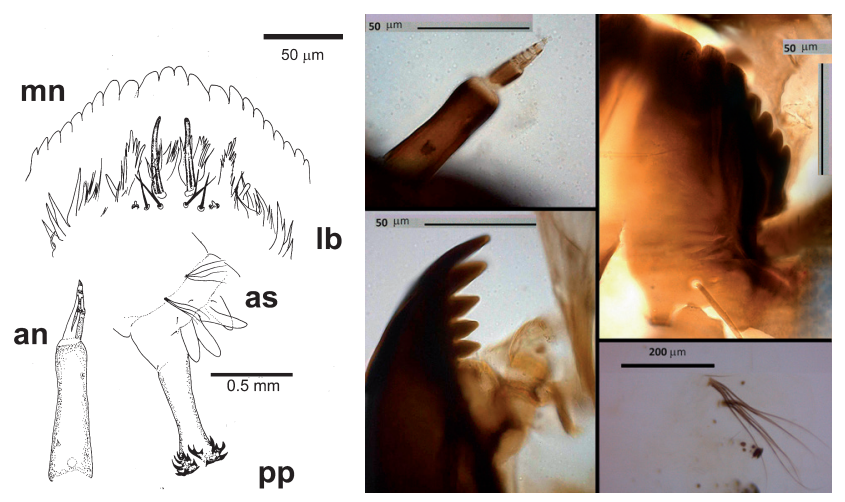

Fig. 8 Left: Diamesa incallida, antenna, mentum, mandible, anal setae; right: Diamesa incallida, an: antenna - lb: labrum - mn: mentum - as: anal setae - pp: posterior parapods.

\section{Diamesa starmachi (Kownacki and Kownacka 1970)}

(Fig. 9)

Body length: $6126 \pm 178(6000-6252, \mathrm{n}=2)$, head capsule yellow, mandibles entirely black, distal margin of mentum and occipital margin with a large black area, head length: $651 \pm 1.58(650-652, \mathrm{n}=2)$, head width: 361 $\pm 0.88(361-362, \mathrm{n}=2)$.

Antenna: AR: $2.01 \pm 0.23(1.84-2.18, \mathrm{n}=2)$, blade length: $25.5 \pm 0.70(25-26, \mathrm{n}=2)$, accessory blade: 19.6 $\pm 0.87(19-20.2, \mathrm{n}=2)$, distance of ring organ from the base of the 1 st antennal segment: $8.25 \pm 0.35(8-8.5, \mathrm{n}=$ $2)$, proximal antennal seta: $27.2 \pm 0.35(27-27.5, \mathrm{n}=2)$, distal antennal seta: $42.2 \pm 0.35(42-42.5, \mathrm{n}=2)$, style: $11.5 \pm 0(11.5-11.5, \mathrm{n}=1)$, style thickness: $2.20 \pm 0.00$ $(2.2-2.21, \mathrm{n}=2), 1$ st antennal segment: $48 \pm 0(48-48$, $\mathrm{n}=1)$, thickness of 1 st antennal segment: $11.2 \pm 0.35$ 
$(11-11.5, \mathrm{n}=2)$, length of antennal segments $2-3-4-5$ : $24 \pm 2.82(22-26, \mathrm{n}=2), 18.3 \pm 0(18.3-18.3, \mathrm{n}=1), 8.93$ $\pm 0(8.93-8.93, \mathrm{n}=1), 6.35 \pm 0(6.35-6.35, \mathrm{n}=1), 5.82 \pm$ $0(5.82-5.82, \mathrm{n}=1)$.

Labrum with simple setae SIII, SII without a large scale at its base, labral lamellae and chaeta media well developed.

Mentum: length: $293 \pm 44.0(262-324, \mathrm{n}=2)$, setae submenti at some distance from the caudal margin of ventromentum, distance from ventromental plates to setae submenti: $51.5 \pm 0(51.5-51.5, \mathrm{n}=1)$, distance between caudal margin of ventromentum and setae submenti: $10.2 \pm 1.19(9.41-11.1, \mathrm{n}=2)$.

Posterior parapods length: $516 \pm 0(516-516, \mathrm{n}=1)$, procercus length: $7.64 \pm 0(7.64-7.64, \mathrm{n}=1)$, procercus diameter: $24 \pm 0(24-24, \mathrm{n}=1)$, anal setae 4 anal setae, anal setae length: $291 \pm 7.71(286-297, \mathrm{n}=2)$, anal setae thickness: $3.8 \pm 0(3.8-3.8, \mathrm{n}=1)$.

Material examined: Avisio stream, 5128882N, 630927E, Vermigliana, 28/12/1978, $2 \mathrm{~L}$ and 5/1/2005, 1 Pe; Noce Bianco TN, 10/7/2012, 2 L.

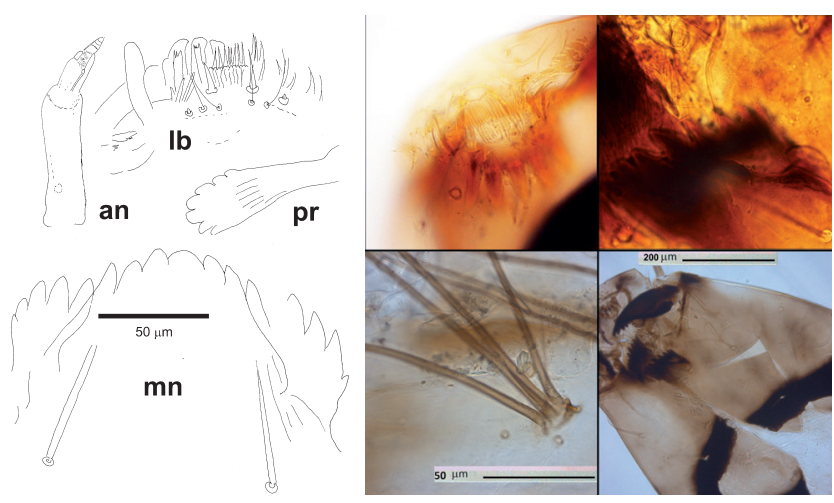

Fig. 9 Left: Diamesa starmachi, labrum, mentum, anal setae, head capsule; right: Diamesa starmachi, an: antenna - lb: labrum - mn: mentum - pr: premandible.

\section{Diamesa zernyi (Edwards 1933); Diamesa vaillanti (Serra-Tosio 1972)}

(Fig. 10)

Body length $8944 \pm 706(7608-9373, \mathrm{n}=10)$, head capsule entirely black, a light area is observable only around eyes, head length: 622. $\pm 21.1(582-633, \mathrm{n}=10)$, head width: $345 \pm 11.7(323-351, \mathrm{n}=10)$.

Antenna: AR: $1.79 \pm 0.20(1.61-2.35, \mathrm{n}=10)$, blade length:29.0 $\pm 2.61(23-31.3, \mathrm{n}=10)$, accessory blade: $16.4 \pm 4.53(8-19.6, \mathrm{n}=10)$, distance of ring organ from the base of the 1st antennal segment: $9.2 \pm 0.78$ (8-10, $\mathrm{n}=10)$, proximal antennal seta: $11.2 \pm 0.78(10-12, \mathrm{n}$ $=10)$, distal antennal seta: $35.2 \pm 0.78(34-36, \mathrm{n}=10)$, style: $8.67 \pm 1.60(7.00-11, \mathrm{n}=10)$, style thickness: $2.23 \pm$ $0.12(2.1-2.46, \mathrm{n}=10), 1$ st antennal segment: $61.0 \pm 2.81$ (54.9-63.1, $\mathrm{n}=10)$, thickness of 1 st antennal segment: $22.8 \pm 0.39(22-23, \mathrm{n}=10)$, combined length of segments 2-5: $34.2 \pm 3.55(25.9-36.5, \mathrm{n}=10)$, length of antennal segments $2-3-4-5: 18.3 \pm 0(18.3-18.3, \mathrm{n}=1), 8.93 \pm 0$
$(8.93-8.93, \mathrm{n}=1), 6.35 \pm 9.36(6.35-6.35, \mathrm{n}=10), 5.82 \pm$ $9.36(5.82-5.82, \mathrm{n}=1)$.

Labrum with bifid setae SIII, SII without a large scale at its base, labral lamellae and chaeta media well developed.

Mandible with 1st lateral tooth more developed.

Mentum: length: $309 \pm 28.7(282-336, \mathrm{n}=10)$, with a paired (or apparently simple but always notched) median tooth and 10 lateral teeth, the 10th very small, distance from ventromental plates to setae submenti: $50.7 \pm 2.04$ (48.8-52.7, $\mathrm{n}=10)$, setae submenti arising anteriorly with respect to caudal extremity of ventromental plates, that is distance between caudal margin of ventromentum and setae submenti is less than $0:-8.5 \pm 3.85(-12--4.8$, $\mathrm{n}=2$ ).

Posterior parapods length: $697 \pm 29.5(678-739, \mathrm{n}$ $=10)$, procercus length: $8.13 \pm 0.21(7.53-8.2, \mathrm{n}=10)$, procercus diameter: $18.3 \pm 0.15(18.2-18.7, \mathrm{n}=10)$, anal setae length: $184 . \pm 74.2(78.5-303, \mathrm{n}=11)$, shorter than in $D$. tonsa, anal setae thickness: $5.87 \pm 0.66(4.98-6.87$, $\mathrm{n}=10)$.

Material examined: upper Ticino river, 5146927N, 1465965E, PiccoloNaret, 20/9/1994, L 56, Lavizzara, 20/9/1994, L 2; Toce river, 5114185N, 1437521E, Bognanco, 12/9/2000, L 42; Paione med, 13/9/2000, L 11; Devero, 4/10/2000, L 1; Sarca river, 5124605N, 1638580E, Alplake SanGiuliano inlet, 23/7/1996, L 1; upper Adda river SO, $5128571 \mathrm{~N}, 1615524 \mathrm{E}$, Adda 31 Sforzell1, 1/10/2013, L 21; Braulio Stelvio, 8/9/2001, ô 1; Adda 38 Mallero3, 24/5/1999, L 1; Adda 38 Mallero, 30/7/1998, ㅇ 1; Braulio Stelvio, 15/8/1977, q Pe 1; Adda 31 Forni, 10/8/1975, L 11; upper Ticino river, 5147220N, 1468824E, Laghetto inf massi, 1/8/1991, L 11; Laghetto inferiore, 1/8/1991, L 44; upper Oglio river, 5121360N, 1606019E, Vezza st 01 monte, 31/3/2014, L 1; Vezza st 01 monte, 4/4/2014, L 1; Vezza st 01 monte, 23/4/2014, L 3; Vezza st 01 monte, 5/9/2014, Pe 1; Grande valle 01 BS, 27/12/2007, Pe 1; Vezza st 02, 28/12/2007, L 1; DezzoD2Valbona, 10/6/1989, L 8; DezzoD1Valbona, 10/6/1989, L 5; Vione, 16/5/1985, L 1; Vezza st 02, 15/2/1980, Le 1; Vezza st 02, 10/4/1980, L 2; S Apoll spring, 20/7/1980, ठ̊ Pe 1; Aviolo alplake, $15 / 8 / 1979$, prep 1; Vione, 10/9/1979, L 4; Vezza st 02, 26/12/1979, L 2; Vezza st 02, 31/12/1979, L 8; Vezza st 02, 26/4/1978, L 1; S Apoll spring, 20/7/1978, ठ̋ Pe 2; Antermoia lake, 5040537N, 1656260E, Antermoia, 26/7/2000, L 19; Avisio stream, 5128882N, 1630927E, Vermigliana, 21/3/2014, Pe 5; Vermigliana, 12/4/2014, L 4; Avisio, 3/7/2000, L 3; Avisio, 4/7/2000, L 365; Avisio, 25/7/2000, L 46; Avisio, 22/8/2000, L 79; Avisio, 23/8/2000, L 15; Bocche, 5040537N, 1656260E, Bocche, 5/7/2000, L 25; Vedretta Carè Alto (car), 5108398N, 1625954E, car 2C7 K, 9/8/2005, L 20; car 1C8 D, 1/6/1997, L 1; car 2C7 C, 1/9/1996, L 6; Dora Baltea river, 5080029N, 1406854E, Lys, 23/6/1987, ô P 1; Dora Ferret glacial stream, 5081210N, 1347345E, Dora Ferret glacial, 4/7/1995, L 3; Ferret gh Pre de Bar, 4/7/1995, $\widehat{\sigma} 1$; Ferret ruscello al- 
pha, 4/7/1995, L 1; Dora Veny glacial stream, 5068650N, 1331469E, Estellette, 8/9/1999, ô 1; Col De La Seigne, 9/8/1998, đo 1; Combal alplake, 8/9/1998, L 1; Mt Blanc, 13/9/1997, ô 2; Estellette, 6/7/1995, ô 1; Combal alplake, 10/9/1995, ㅅ 1; Lambin, 5107511N, 1622276E, Alplake Lambin in, 7/8/1996, L 1; Lambin, 5107511N, 1622276E, Alplake Lambin ou, 7/8/1996, L 9; Sesia river, 5079954N, 1412029E, Olen stream, 15/9/1990, Pe 1; Parco Naz. Gran Paradiso (PNGP) Orco river, 5030436N, 1361018E, F2ROC-1, 9/7/2010, L 33; Sarca river, 5107511N, 1622276E, Alplake Ritorto in, 18/6/1996, L 1; Tovel, 5123708N, 1647748E, Tovel alplake, 7/8/1996, P 11; Marmore glacial stream, $5087560 \mathrm{~N}, 1393950 \mathrm{E}$, Cervinia, 21/9/1980, L 3; Orta lake, 5073949N, 1453385E, Orta, 21/11/1992, L 11; Cherio river, Endine lake, 5070494N, 1572555E, EndineG01ql, 8/9/1979, L 11; Brembo river, 5082151N, 1559099E, Ola Alben Oltre il Colle, 5/12/1982, §ิ 1; 5090635N, 1549072E, Brembo01Cassiglio, 23/1/1980, L 1; 5098159N, 1561912E, Brembo01Carona, 23/5/1980, L 1; 5092431N, 1541312E, Brembo00Valtorta, 25/2/1979, L 1; Brembo00Valtorta, 4/6/1979, L 1; Lambro river, 5071949N, 1518133E, Lamb06Caslino, 2/3/2004, Pe 7; Lamb06Caslino, 29/10/2004, L 1; Lamb01Magreglio, 27/12/1976, L 1; Varese lake, 5075605N, 1478529E, Varese lake Gavirate, 23/4/1998, L 1; Varese lake Schiranna, 23/4/1969, L 1; Varese lake Gavirate, 23/4/1920, L 1; Varese lake Schiranna, 23/4/1906, Pe 1; Bormida river, 4896408N, 1429434E, Bormida, 23/4/1988, L 1; Quiliano, 4903589N, 1454783E, Dane stream, 15/3/1989, $q$ 1; Aveto stream, 4927581N, 1532286E, Aveto applake, 16/4/2009, L 2.

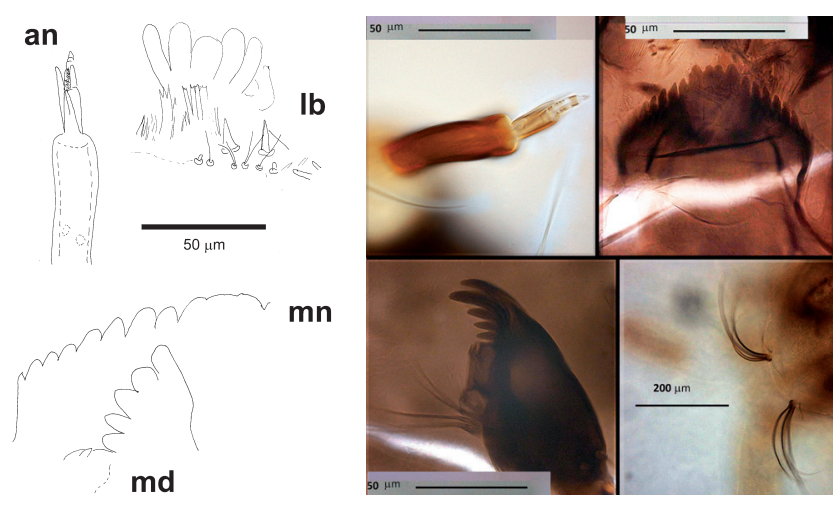

Fig. 10 Left: Diamesa zernyi, antenna, mentum, mandible, caudal end/anal setae; right: Diamesa zernyi, an: antenna - lb: labrum - mn: mentum - md: mandible.

\section{Diamesa insignipes (Kieffer in Kieffer and Thienemann 1908)}

(Fig. 11)

Body length $7436 \pm 25.8(7427-8000, \mathrm{n}=8)$, head capsule yellow with reduced paired brown spots in frontalapotome and in genae, occipital margin and mandible black, anterior margin of mentum black, the color of mentum gradually lighten until becoming yellow in caudally, premandible black, first antennal segment black, but lightens at its base, head length: $749 \pm 9.40$ (727-756, $\mathrm{n}=8)$ head width: $416 \pm 5.22(403-420, \mathrm{n}=8)$.

Antenna: AR: $1.75 \pm 0.18(1.43-2.01, \mathrm{n}=8)$. The an- tennal blade reach the base of the 4 th segment, blade length $27 \pm 1.31(25.5-28.3, \mathrm{n}=8)$ the accessory blade reach the basal third of the 3rd segment: $20.1 \pm 1.27$ $(18.5-21.1, \mathrm{n}=8)$ distance of ring organ from the base of the 1 st antennal segment: $11 \pm 0.75(10-12, \mathrm{n}=8)$ proximal antennal seta: $13 \pm 0.75(12-14, \mathrm{n}=8)$ distal antennal seta: $34 \pm 0.75(33-35, \mathrm{n}=8)$ style: $9.06 \pm 0.97(7.93-9.99$, $\mathrm{n}=8)$ style thickness: $2.13 \pm 0.07(2.1-2.3, \mathrm{n}=8) 1$ st antennal segment: $61.3 \pm 4.50(54.6-68.5, \mathrm{n}=8)$ thickness of 1 st antennal segment: $19.9 \pm 1.86(18-22, \mathrm{n}=8)$, combined length of segments 2-5: $35.1 \pm 3.84(31.1-42.5, \mathrm{n}=$ $8)$, lengths of segments $2-3-4-5: 18.3 \pm 0(18.3-18.3, \mathrm{n}=$ $8), 8.93 \pm 0(8.93-8.93, \mathrm{n}=8), 6.35 \pm 9.49(6.35-6.35, \mathrm{n}=$ $8), 5.82 \pm 9.49(5.82-5.82, \mathrm{n}=8)$.

Labrum with bifid setae SIII, SII without a large scale at its base, labral lamellae and chaeta media well developed.

Mentum: length: $306 \pm 30.9(273-340, \mathrm{n}=8)$, with 11 lateral teeth and a single median tooth, but it appears a bit notched, distance from ventromental plates to setae submenti: $50.3 \pm 4.52(47.0-57.5, \mathrm{n}=8)$, setae submenti arising anteriorly with respect to caudal extremity of ventromental plates, that is distance between caudal margin of ventromentum and setae submenti is less than $0:-5.9$ $\pm 7.11(-10--6.20, \mathrm{n}=2)$

Posterior parapods length: $516 \pm 0(516-516, \mathrm{n}=8)$ about $7 \%$ of body length, procercus length: $8.16 \pm 0.38$ (7.53-8.37, $\mathrm{n}=8)$, procercus diameter: $21.0 \pm 1.43$ (18.7-21.8, $\mathrm{n}=8)$, anal setae length: $291 \pm 42.1$ $(207-337, \mathrm{n}=8)$, anal setae thickness: $6.16 \pm 0.92$ $(4.45-6.86, \mathrm{n}=8)$.

Material examined: Lambro river, $5075670 \mathrm{~N}$, 1523301E, Lamb04Asso, 21/6/1978, L 1; Bormida river, 4896408N, 1429434E, Calizzano Mereta A2, 22/1/1990, L 1; Monesiglio C9, 22/1/1990, L 1; Millesimo Acqua Fredda C1, 22/1/1990, L 2; Calizzano Caragna A3, 22/1/1990, L 3; Murialdo Isoletta A5, 12/3/1990, L 2; Calizzano Caragna A3, 12/3/1990, L 4; Calizzano Mereta A2, 1/5/1990, L 1; Calizzano Mereta A2, 12/6/1990, L 2; Osiglia, 12/6/1990, L 1; Osiglia, 23/7/1990, L 1; Murialdo Isoletta A5, 20/3/1989, L 12; Millesimo nord C3, 20/3/1989, L 4; Calizzano Mereta A2, 18/4/1989, L 2; Murialdo Isoletta A5, 18/4/1989, L 7; Calizzano Mereta A2, 15/5/1989, L 23; Murialdo Isoletta A5, 15/5/1989, L 13; Millesimo nord C3, 15/5/1989, L 2; Cengio presa ACNA C4, 15/5/1989, L 7; Calizzano Caragna A3, 15/5/1989, L 8; Calizzano Mereta A2, 24/7/1989, L 25; Murialdo Isoletta A5, 24/7/1989, L 1; Osiglia, 24/7/1989, L 2; Calizzano Caragna A3, 24/7/1989, L 3; Calizzano Mereta A2, 20/9/1989, L 6; Osiglia, 20/9/1989, L 1; Calizzano Caragna A3, 7/11/1989, L 2; Lavagna, 4907190N, 1526583E, Lavagna, 21/2/1980, L 1; Taro river, 4929000N, 1552000E, Taro 2, 4/3/2003, L 2; Taro 2, 10/1/2002, ठ Pe 100; Taro 3, 10/1/2002, ô 2; Taro 2, 1/3/2002, Pe 26; Taro 2, 26/3/2002, Pe 1; Taro 3, 24/4/2001, L 25; Stirone, 4961690N, 1605457E, Stirone Parma, 18/1/1977, prep 1; Trebbia, 4924221N, 1574244E, Cisa, 6/3/1981, ô Pe 1; 
Brasimone artificial lake, 4888985N, 1670664E, Brasimone, 20/3/1990, § 1; Brasimone, 4888985N, 1670664E, Brasimone, 22/3/1990, స 1; Sangro river, 4638802N, 1898838E, PNA01, 19/5/1978, L 6; PNA11CastelSangro, 20/5/1978, ふ̋ 1; PNA04, 21/5/1978, L 1; PNA12, 21/5/1978, L 1; Tordino stream, 4740698N, 1894453E, Tord09, 24/11/1989, L 4; Calore, 4493183N, 2034680E, Valle AngeloR10, 10/3/2001, L 1; Calore, 4493183N, 2034680E, LaurinoR10, 3/10/2000, L 4; Ripiti, 4493183N, 2034680E, RipitiP12, 9/3/2001, L 1; RipitiP15, 9/3/2001, L 3; RipitiR01, 9/3/2001, L 3; RipitiR02, 9/3/2001, L 6; RipitiR06, 9/3/2001, L 5; RipitiR08, 9/3/2001, L 1; RipitiR10, 9/3/2001, L 1; Sammaro, 4493183N, 2034680E, Sammaro refP01, 27/5/2000, L 1; Sammaro refP02, 27/5/2000, L 1; Sammaro refP03, 27/5/2000, L 4; Sammaro refP05, 27/5/2000, L 15; Sammaro refP06, 27/5/2000, L 1; Sammaro refP07, 27/5/2000, L 1; Sammaro refR11, 27/5/2000, L 3; Sammaro refR12, 27/5/2000, L 1; Sammaro refR15, 27/5/2000, L 1; Sammaro refR16, 27/5/2000, L 1; Sammaro refR20, 27/5/2000, L 10; Sassano, 4485896N, 2062270E, Sassano valleR02, 13/3/2001, L 1; Tanagro, $4517256 \mathrm{~N}, 2032953 \mathrm{E}$, Tanagro refR03, 12/3/2001, L 1; Tanagro refR08, 12/3/2001, L 1; AscoleseR06, 13/3/2001, L 1; Tanagro refR01, 13/10/2000, L 1; Torno, 4441682N, 2016632E, Torno valleR01, 9/3/2001, L 7; Torno valleR02, 9/3/2001, L 9; Torno valleR03, 9/3/2001, L 1.
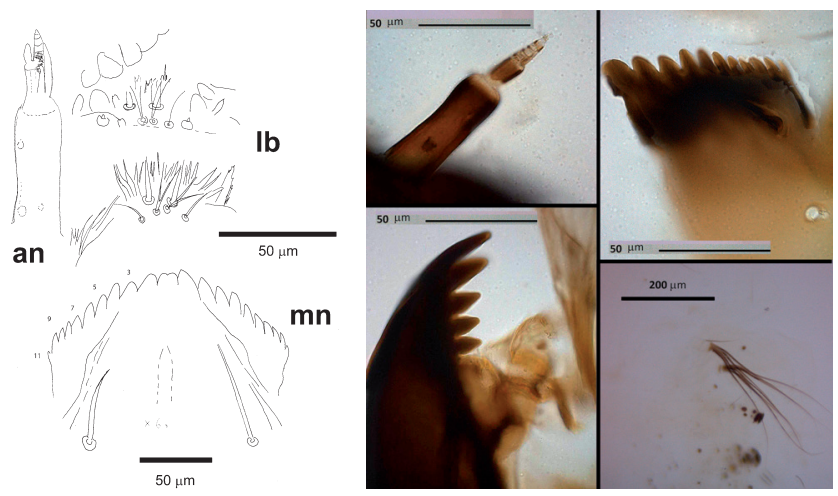

Fig. 11 Left: Diamesa insignipes, antenna, mentum, mandible, anal setae; right: Diamesa insignipes, an: antenna - lb: labrum - mn: mentum.

\section{Diamesa cinerella (Meigen in Gisti 1835)}

(Fig. 12)

Body length: $11140 \pm 102(11000-11209, \mathrm{n}=7)$, genrally larger than similar species, head capsule entirely yellow except tips of mandibles and mentum. Head length: $1063 \pm 111(900-1128, \mathrm{n}=7)$, head width: $590 \pm 61.9$ $(500-626, \mathrm{n}=7)$.

Antenna: AR: $1.91 \pm 0.17(1.61-2.16, \mathrm{n}=7)$, blade length:31.1 $\pm 0.31(31.0-31.8, \mathrm{n}=7)$, accessory blade: 21.6 $\pm 0.41(21.3-22.3, n=7)$, distance of ring organ from the base of the 1 st antennal segment: $13.0 \pm 0.04(13-13.1$, $\mathrm{n}=7)$, proximal antennal seta: $11.0 \pm 0.04(11-11.1$, $\mathrm{n}=7)$, distal antennal seta: $38.0 \pm 0.04(38-38.1, \mathrm{n}=7)$, style: $8.27 \pm 0.93(6.67-9.27, \mathrm{n}=7)$, style thickness: 2.35 $\pm 0.07(2.3-2.5, \mathrm{n}=7)$, 1st antennal segment: $66.4 \pm 6.82$ (55.6-76.2, $\mathrm{n}=7)$, thickness of 1 st antennal segment:
$18.4 \pm 0.80(17-19, \mathrm{n}=7)$, combined length of segments $2-5: 34.6 \pm 1.11(33.5-36.7, \mathrm{n}=7)$, length of antennal segments 2-3-4-5: $18.3 \pm 0(18.3-18.3, \mathrm{n}=7), 8.93 \pm 0$ $(8.93-8.93, \mathrm{n}=7), 6.35 \pm 9.59(6.35-6.35, \mathrm{n}=7), 5.82 \pm$ $9.59(5.82-5.82, \mathrm{n}=7)$.

Labrum with bifid setae SIII, SII without a large scale at its base, labral lamellae and chaeta media well developed.

Mentum: length: $379 \pm 152(281-602, \mathrm{n}=7)$, with a simple median tooth sometime with a small notch and (8)-9 lateral teeth, distance from ventromental plates to setae submenti: $51.8 \pm 1.58(49-52.7, \mathrm{n}=7)$, setae submenti arising anteriorly with respect to caudal extremity of ventromental plates, that is distance between caudal margin of ventromentum and setae submenti is less than 0: $-4.1 \pm 8.45(-7.3--15, \mathrm{n}=2)$.

Posterior parapods length: $656 \pm 167(446-787, \mathrm{n}=7)$, procercus length: $11.0 \pm 1.90(9.01-12.5, \mathrm{n}=7)$, procercus diameter: $19.4 \pm 3.07(17-22.7, \mathrm{n}=7)$, anal setae length: $282 \pm 18.4(268-316, \mathrm{n}=7)$, anal setae thickness: $5.54 \pm 0.59(4.71-6.32, \mathrm{n}=7)$.

Material examined: Sarca river, 5124605N, 1638580E, Alplake SanGiuliano in, 10/8/1996, L 1; upper Adda river SO, 5141560N, 1616559E, Adda 31 Forni, 21/8/2013, L 1; Adda 38 Mallero1b, 6/11/1998, L 4; Braulio Stelvio, 14/4/1977, PeF 1; Avisio stream, 5120251N, 1804943E, TN 17 Valda, 15/3/1999, L 100; upper Oglio river, 5128571N, 1615524E, S Apoll ruscello, 21/3/2014, L 30; S Apoll spring, 2/1/1981, L 1; Aviolo alplake, 9/8/1979, L 1; Vezza st 02, 25/3/1978, L 4; Vezza st 02, 10/8/1978, §ิ P 2; Avisio stream, 5128882N, 1630927E, Vermigliana, 16/2/1981, L 1; Vermigliana, 25/2/1980, đ̂ 1; Vermigliana, 22/4/1978, L 1; CimaArtuich, 5107511N, 1622276E, Alplake Artuich in, 10/8/1996, L 1; Lys glacial stream, 5092954N, 1409631E, Gressoney, 1/7/1987, đ̂ 1; Sarca river, 5105960N, 1619818E, NuovoAdamBrenta, 22/8/1996, $\mathrm{P}+$ đ̄ 6; Olona river, 5068303N, 1467600E, RasaQ39, 27/2/1997, đ̊ 1; Tagliamento, 5120251N, 1804943E, Tagliamento, 0/3/1999, L 3; Tagliamento, 15/3/1999, L 5; Tgl1 5 1, 15/3/1999, L 3; Tgl1 5 3O, 15/3/1999, L 14; Tgl1 5 5, 15/3/1999, L 15; TglII ô 2, 15/3/1999, L 70; TglII ô 4, 15/3/1999, L 36; TglIV ô 2, 15/3/1999, L 4; TglIV ô Q, 15/3/1999, L 4; Alserio lake, 5072588N, 1515002E, Alserio lake 0, 17/12/2004, Pe 1; Alserio 4, 17/12/2004, Pe 1; Pioverna, 5088695N, 1536169E, Pioverna stream, 17/1/2002, Pe 2; Pioverna stream, 8/2/2002, L 1; Adda river BG, 5046136N, 1541620E, Vaprio, 22/2/2002, Pe 2; Adda river CR, 5035927N, 1537780E, Muzza Rivolta CR, 24/1/2002, Pe 14; Muzza Rivolta CR, 22/2/2002, Pe 5; Muzza Rivolta CR, 9/3/2002, Pe 2; Adda river LO, 5016501N, 1540510E, Lodi, 22/2/2002, Pe 5; Comazzo, 28/3/2002, Pe 2; Ticino river MI, 5010292N, 1500741E, Ticino Bereguardo, 23/2/2002, đิ P 1; Ticino Boffalora, 4/3/1993, L 1; Ticino Boffalora, 16/12/1979, q Pe 1; Trebbia, 4924221N, 1574244E, Cisa, 5/3/1981, L 20; Cisa, 3/5/1981, ふै 2 . 


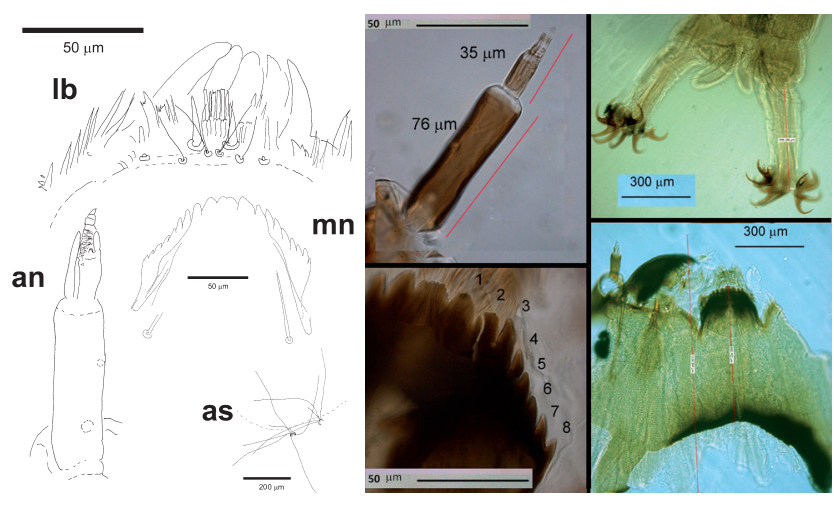

Fig. 12 Left: Diamesa cinerella, antenna, caudal end/posterior parapods, mentum, head capsule; right: Diamesa cinerella, an: antenna - lb: labrum - mn: mentum - hc: head capsule.

\section{Diamesa tonsa (Haliday in Walker 1856)}

(Fig. 13)

Body length: $9335 \pm 118$ (9000-9373, $\mathrm{n}=10)$, head capsule with extended brown-black areas, head length: $555 \pm 42.0$ (495-582, $\mathrm{n}=10)$, head width: $308 \pm 23.3$ $(275-323, \mathrm{n}=10)$.

Antenna: the antennal blade reaches the distal margin of 4th segment, AR: $1.84 \pm 0.17(1.45-2.06, \mathrm{n}=10)$, blade length: $28.6 \pm 2.25(25-31.9, \mathrm{n}=10)$, accessory blade: $17.6 \pm 1.86(16-21.3, \mathrm{n}=10)$, distance of ring organ from the base of the 1 st antennal segment: $12.8 \pm 3.11$ (7-16, $\mathrm{n}=10)$, proximal antennal seta: $10.8 \pm 2.16(6.8-12, \mathrm{n}=$ $10)$, distal antennal seta: $43.8 \pm 4.99(38.1-53, \mathrm{n}=10)$, style: $9.13 \pm 1.02(8-10.3, \mathrm{n}=10)$, style thickness: $2.38 \pm$ $0.07(2.3-2.5, \mathrm{n}=10)$, 1st antennal segment: $69.4 \pm 7.15$ $(58-80, \mathrm{n}=10)$, thickness of 1 st antennal segment: 19.2 $\pm 3.74(19.2-19.2, \mathrm{n}=10)$, combined length of segments $2-5: 37.7 \pm 2.56(33.5-40, \mathrm{n}=10)$, length of antennal segments $2-3-4-5: 18.3 \pm 0(18.3-18.3, \mathrm{n}=1), 8.93 \pm 0$ $(8.93-8.93, \mathrm{n}=1), 6.35 \pm 0(6.35-6.35, \mathrm{n}=1), 5.82 \pm 0$ $(5.82-5.82, \mathrm{n}=1)$,

Labrum with bifid setae SIII, SII without a large scale at its base, labral lamellae and chaeta media well developed.

Mentum: length: $291 \pm 14(277-304, \mathrm{n}=10)$, brown also in caudal part, with a single median tooth (sometime notched) and 9 lateral teeth, sometime a very small 10th tooth is present, distance from ventromental plates to setae submenti: $52.2 \pm 1.06(50.1-52.7, \mathrm{n}=10)$, setae submenti arising anteriorly with respect to caudal extremity of ventromental plates, that is distance between caudal margin of ventromentum and setae submenti is less than $0:-7.7 \pm 0.62(-8.6--7.3, \mathrm{n}=2)$.

Posterior parapods length: $542 \pm 12.7(527-552, \mathrm{n}=$ 10), procercus length: $9.01 \pm 0(9.01-9.01, \mathrm{n}=1)$, procercus diameter: $22.7 \pm 3.74(21.1-25.7, \mathrm{n}=3)$, anal setae length: $330 \pm 47.7(300-400, \mathrm{n}=10)$, anal setae thickness: $5.30 \pm 0.66(4.24-6.32, \mathrm{n}=10)$.

Material examined: upper Adda river SO, 5129784N, 1574426E, Adda 38 Bignami, 12/8/1993, Pe 1; Adda 01 Viola, 0/8/1985, ㅇ Pe 1; Adda 01 Viola, 0/9/1985, ô 1; upper Oglio river, 5121534N, 1605373E, Vezza st 02,
11/3/2014, L 1; S Apoll spring, 4/4/2014, L 1; S Apoll ruscello, 11/4/2014, ô 1; Vezza st 01 monte, 1/9/2014, Pe 1; Dezzo08, 0/6/1989, L 1; DezzoA1, 0/6/1989, L 20; DezzoA2, 0/6/1989, L 9; DezzoD6Valbona, 0/6/1989, L 3; DezzoD7Valbona, 0/6/1989, L 2; DezzoE1, 0/6/1989, L 11; DezzoF1, 0/6/1989, L 50; DezzoF3, 0/6/1989, L 1; Oglio alta valle Camonica, 0/6/1989, L 1; Vezza st 02, 0/7/1989, đ̋ Pe 1; Vezza st 02, 12/3/1988, Pe 1; Vione, 0/6/1985, L 1; Vione, 0/8/1985, Pe 1; Vione, 0/9/1985, L 1; Vione, 0/11/1985, L 3; Vione, 20/4/1981, L 2; DezzoA1, 0/6/1981, L 1; Vezza st 02, 15/0/1980, 9 1; Vezza st 02, 1/1/1980, L 1; Frigidolfo, 15/2/1980, Pe 1; Vezza st 02, 24/2/1980, L 1; Vezza st 02, 0/8/1980, Pe 1; Vezza st 02, 20/5/1979, L 5; Vezza st 02, 0/7/1979, ô P 1; Vezza st 02, 4/8/1979, L 31; Vezza st 02, 5/8/1979, L 16; Vezza st 02, 19/8/1979, L 78; Vezza st 02, 4/3/1978, L 6; Vezza st 03, 25/3/1978, L 3; S Apoll spring, 25/4/1978, L 1; Temu', 27/4/1978, L 2; Vezza st 02, 20/5/1978, L 5; Vezza st 02, 3/7/1978, đ̂ 1; Vezza st 02, 10/7/1978, L 5; Vezza st 03, 22/7/1978, L 7; Vezza st 02, 22/7/1978, L 1; Vezza st 02, 23/7/1978, L 2; Vezza st 02, 22/8/1978, L 1; Vezza st 02, 14/11/1978, L 1; Avisio stream, 5128882N, 1630927E, Vermigliana, 4/4/2014, L 1; Vermigliana, 9/5/2014, L 20; Vermigliana, 23/2/2007, Pe 1; Vermigliana, 15/2/1980, Pe 1; Vermigliana, 16/4/1978, त̂ 1; Dora Veny glacial stream, 5068650N, 1331469E, Combal alplake, 6/7/1995, ô 1; Miage, 10/9/1995, Pe 1; Lambin, 5107511N, 1622276E, Alplake Lambin ou, 10/8/1996, L 9; Sarca di Genova river, 5107511N, 1622276E, Alplake Mandrone in, 10/8/1996, L 6; Mandrone, 10/8/1996, L 33; Negro lake Gavia pass, 5134079N, 1612854E, Gavia passo lago Negro, 3/7/1978, L 22; Parco Naz. Gran Paradiso (PNGP) Orco river, 5030436N, 1361018E, F2-ROC-2B, 9/7/2010, L 153; F2-ROC-2B, 18/8/2010, L 66; F2-ROC-1, 19/8/2010, L 4; F2-ROC-2A, 19/8/2010, L 4; Sarca river, 5107511N, 1622276E, Alplake Ritorto in, 10/8/1996, L 1; Ritorto, 10/8/1996, L 22; Sarca river, 5124605N, 1638580E, TreLaghiPrincipale, 10/8/1996, L 6; Serodoli, 5107511N, 1622276E, Alplake Serodoli in, 10/8/1996, L 1; Tonale, $5123166 \mathrm{~N}, 1623338 \mathrm{E}$, Tonale passo, 23/2/2007, Pe 1; TreLaghiI, 5124605N, 1638580E, Tre Laghil, 10/8/1996, L 15; Venerocolo, 5115584N, 1614483E, Venerocolo 01 alplake, 23/8/1978, L 19; Orta lake, Acqualba, Lagna stream, $5077694 \mathrm{~N}, 1448237 \mathrm{E}$, Acqualba02, 27/3/1976, L 2; Acqualba02, 1/5/1976, L 4; Acqualba03, 1/5/1976, L 2; Acqualba03, 30/11/1976, L 1; Acqualba04, 15/12/1976, L 1; Elvo, 5044666N, 1419337E, Elvo Sordevolo, 5/5/2001, ô Pe 3; Elvo, 5044666N, 1419337E, Elvo Sordevolo, 11/5/2001, ổ Pe 1; Agogna, Grua stream, 5066562N, 1450737E, Grua04, 1/1/1978, L 2; Grua01, 1/3/1977, L 8; Grua02, 1/3/1977, L 50; Grua03, 0/4/1977, L 1; Grua01, 1/4/1977, L 3; Grua02, 1/4/1977, L 11; Grua04, 1/4/1977, L 3; Grua05, 1/4/1977, L 2; Grua03, 0/5/1977, L 1; Grua02, 1/5/1977, L 70; Grua04, 1/5/1977, L 2; Grua03, 5/6/1977, L 1; Grua03, 1/12/1977, L 11; Tagliamento, 5120251N, 1804943E, TglV 1 2reach, 3/5/1998, P 1; Tagliamento, 5168090N, 
1831995E, Carnia, 0/6/1980, L 1; Carnia, 17/6/1980, ô P 1; AnnoneEst, 5071529N, 1527036E, Annone Est, 3/4/2004, Pe 1; Annone est 1950 2004, 3/4/2004, Pe 1; Bardello, 5075670N, 1476700E, Bardello 03, 1/3/1979, ㅇ P 1; Como, 5075660N, 1520712E, Como Lecco, 3/4/2004, Pe 1; Como, 3/4/2004, Pe 1; Cherio river, Endine lake, 5070494N, 1572555E, EndineG01ql, 25/2/1981, L 11; EndineG01qt, 25/2/1981, L 22; EndineG01qt, 1/4/1981, L 11; EndineG03qt, 18/9/1980, L 11; Garlate, 5073074N, 1531750E, Garlate lake, 3/4/2004, Pe 2; Garlate, 3/4/2004, Pe 2; Ghirla, 5079548N, 1479539E, Ghirla lake 1, $11 / 3 / 1977$, L 1; Brembo river, 5062386N, 1551848E, Brembo Parco Colli, 14/3/2007, Pe 2; Brembo Parco Colli, 14/9/2007, Pe 1; Brembo01Carona, 22/6/2003, Pe 3; Brembo allev 1802 01, 13/4/2001, đ̊ Pe 1; Brembo01Carona, 17/6/1980, Pe 1; Lambro river, 5068245N, 1518144E, Lamb08Merone, 27/1/2005, L 5; Lamb11Carate, 2/3/2004, L 3; Lamb08Merone, 2/3/2004, L 8; Lamb11Carate, 29/3/2004, † 1; Lamb08Merone, 29/3/2004, L 6; Lamb06Caslino, 29/4/2004, L 6; Lamb08Merone, 29/4/2004, L 1; Lamb08Merone, 26/11/2004, L 1; Lamb09Lambrugo, 8/2/1996, L 1; Lamb05SCalocero, 8/2/1996, L 1; Lamb06Caslino, 15/2/1996, L 24; Lamb11Carate, 15/3/1996, L 1; Lamb08Merone, 23/3/1996, L 2; Lamb02Barni, 23/3/1996, L 7; Lamb04Asso, 23/3/1996, L 2; Lamb03Lasnigo, 18/4/1996, L 1; Lamb02Barni, 17/5/1996, L 1; Lamb13Brugherio, 21/5/1996, L 1; Lamb13Brugherio, 16/7/1996, L 1; Lamb09Lambrugo, 28/10/1996, L 1; Lamb09Lambrugo, 26/1/1978, L 1; Lamb05SCalocero, 10/3/1978, L 1; Lamb03Lasnigo, 10/3/1978, L 1; Lamb04Asso, 10/3/1978, L 1; Lamb09Lambrugo, 3/2/1977, L 20; Lamb01Magreglio, 3/2/1977, L 8; Lamb02Barni, 3/2/1977, L 44; Lamb03Lasnigo, 3/2/1977, L 58; Lamb01Magreglio, 27/12/1977, L 1; Lamb04Asso, 10/3/1976, L 1; Lamb06Caslino, 11/6/1976, L 1; Lamb05SCalocero, 11/11/1976, L 1; Lamb01Magreglio, 11/11/1976, L 1; Lamb02Barni, 11/11/1976, L 19; Lamb03Lasnigo, 11/11/1976, L 10; Lamb05SCalocero, 22/11/1976, L 1; Lamb03Lasnigo, 22/11/1976, L 10; Lamb02Barni, 30/11/1976, L 19; Lamb04Asso, 0/12/1976, L 1; Lugano, 5090441N, 1498709E, Lugano lake, 8/4/2004, Pe 9; Lugano lake, 8/4/2003, Pe 9; MaggioreVA, 5093885N, 1478585E, Magg 03sGermignaga, 30/4/1978, L 1; Piano, 5098262N, 1512532E, Piano lake 1, 8/4/2004, Pe 1; Pioverna, 5092346N, 1525820E, Pioverna stream, 3/2/1976, Pe 7; Pusiano, 5072206N, 1521220E, Pusiano 4, 29/3/2004, Pe 1; Pusiano, 29/3/2004, Pe 1; Pusiano 2, 0/0/1800, L 1; Serio, 5050363N, 1557875E, Basella di Urgnano, 30/4/2003, Pe 1; Ghisalba, 20/12/2003, Pe 1; Ricengo, 23/1/2000, Pe 2; Vivione passo, 0/7/1979, L 1; Vivione passo, 10/9/1979, L 1; TicinoNO, 5051618N, 1471411E, Ticino Oleggio, 22/3/2004, Pe 35; Ticino Oleggio, 27/4/2004, Pe 1; Ticino Oleggio, 18/3/2003, Pe 5; Ticino Oleggio, 15/4/2003, Pe 2; Vareselake, 5074179N, 1478564E, Varese lake, 3/4/2009, L 15; Brenta, 5059757N, 1681765E, Brenta, 23/7/1977, § 1; GardaBS, 5038209N,
1626354E, Garda 19bManerba, 19/3/2004, Pe 4; GardaVR, 5032590N, 1632978E, Garda 26vPeschiera, 19/3/2004, Pe 4; Idro, 5065704N, 1611758E, Idro lake, 23/4/2004, Pe 2; OglioBS, 5061720N, 1606399E, Chiese, 14/4/1994, Pe 30; Agogna stream, 5004753N, 1471743E, Zeme Zanaglia canale, 26/2/2010, Pe 1; Agogna 89, 3/3/1989, L 1; Agogna 03 Cavaglio Tesi Zanetti, 30/4/1981, L 1; Agogna 04NO, 3/6/1981, L 1; Agogna 07 $\mathrm{NV}=$ staz 4 valle Novara, 3/6/1981, L 3; Agogna 06 NS=staz 3 (SS n II) Novara sud, 3/6/1981, L 12; Agogna 04NO, 6/6/1981, L 1; Agogna 02 Gozzano Tesi Zanetti, 6/6/1981, L 2; Agogna 04NO, 9/7/1981, L 8; Agogna 03 Cavaglio Tesi Zanetti, 25/7/1981, L 1; Agogna 02 Gozzano Tesi Zanetti, 25/7/1981, L 1; Agogna 05 NO=staz 2Novara ovest, 18/11/1981, L 10; Agogna 03 Cavaglio Tesi Zanetti, 21/11/1981, L 16; Bormida river, 4913001N, 1436254E, Millesimo nord C3, 22/1/1990, L 2; S Michele Saliceto C7, 12/3/1990, L 11; Camerana C8, 12/3/1990, L 27; Gorzegno C11, 12/3/1990, L 1; Millesimo Acqua Fredda C1, 12/3/1990, L 13; Millesimo nord C3, 12/3/1990, L 6; Cengio presa ACNA C4, 12/3/1990, L 4; Pianrocchetta C6, 12/3/1990, L 1; Bormida, 1/4/1990, L 1; Bormida, 11/4/1990, L 1; Murialdo Isoletta A5, 1/5/1990, L 5; Camerana C8, 1/5/1990, L 1; Osiglia, 1/5/1990, L 1; Cengio presa ACNA C4, 1/5/1990, L 12; Murialdo Isoletta A5, 12/6/1990, L 1; Millesimo Acqua Fredda C1, 12/6/1990, L 2; Calizzano Caragna A3, 12/6/1990, L 1; Millesimo Acqua Fredda C1, 23/7/1990, L 4; Cengio presa ACNA C4, 20/3/1989, L 1; S Michele Saliceto C7, 15/5/1989, L 1; Gorzegno C11, 15/5/1989, L 1; Pianrocchetta C6, 15/5/1989, L 1; Millesimo Acqua Fredda C1, 20/9/1989, L 5; Calizzano Caragna A3, 20/9/1989, L 4; Bormida, 30/1/1988, L 1; Bormida, 12/3/1988, q 1; Bormida, 26/3/1988, L 1; Bormida, 7/5/1988, L 1; Bormida, 13/7/1987, 1; Sciusa, 4909109N, 1460136E, Sciusa, 1/3/1981, L 1; Adda river BG, 5046136N, 1541620E, Vaprio, 24/4/2003, Pe 6; Adda river CR, 5035018N, 1540391E, Rivolta CR, 4/12/2001, P 1; Adda river CR, 5035472N, 1539085E, Rivolta CR, 13/3/1986, L 1; Aveto stream, 4923842N, 1523864E, Agoraie, 5/6/1988, L 1; Sestri, 4899815N, 1533267E, Sestri, 21/9/1988, L 1; Taro river, 4926744N, 1546642E, Taro 1, 26/3/2003, Pe 1; Taro 2, 26/3/2003, Pe 2; Taro 2, 4/3/2002, L 1; Taro 1, 12/3/2002, L 4; Taro 1, 26/3/2002, Pe 100; Taro 1, 23/4/2002, P 2; Taro 2, 23/4/2002, PLe 55; Ceno stream, 14/5/2002, L 1; Taro 2, 15/5/2002, Pe 35; Taro 2, 16/5/2002, Pe 32; Taro 1, 4/6/2002, Pe 1; Taro 2, 24/4/2001, Pe 2; Taro 2, 15/5/2001, Pe 4; Ticino river MI, 5039474N, 1478207E, Ticino Turbigo, 22/3/2004, Pe 2; Ticino Castelletto, 27/4/2004, Pe 3; Ticino Castelletto, 29/1/2003, Pe 2; Ticino Turbigo, 12/3/2003, Pe 5; Ticino Castelletto, $1 / 2 / 2001$, PeM 1; Ticino Castelletto, 5/2/2001, ㅇ Pe 2; Ticino Castelletto, 13/2/2001, ô Pe 1; Ticino Castelletto, 14/2/2001, đ̂ Pe 2; Ticino Castelletto, 15/2/2001, đ̊ Pe 1; Ticino Castelletto, 19/2/2001, ㅇ Pe 1; Ticino Castelletto, 21/2/2001, q Pe 1; Ticino Castelletto, 23/2/2001, Pe 1; Ticino Castelletto, 5/3/2001, q Pe 1; 
Ticino Boffalora, 25/4/1985, ô 1; Po river PC, 4996980N, 1611433E, Po river Piacenza, 2/12/1980, L 1; PoTO, 4961648N, 1397179E, Po river Torino, 14/6/1989, L 2; PoVC, 4996490N, 1421341E, Trino Vercellese, 13/2/1981, P 1; Trino Vercellese, 25/5/1981, P 1; Rosso Trino, 11/11/1981, L 1; Trino Vercellese, 11/11/1981, P 1; Taro river, $4948901 \mathrm{~N}, 1585500 \mathrm{E}$, Taro 4, 26/3/2002, L 1; Taro 3, 2/5/2000, Pe 14; Magra, 4890883N, 1573290E, Magra, 5/3/1981, L 1; Tevere river RM, 4627636N, 1781325E, Castel Porziano, 8/6/1990, L 1; Amaseno, 4599577N, 1859132E, Amasen04, 6/5/1990, L 1; Aso stream, 4771479N, 1880420E, Aso03, 17/4/1980, L 2; Aso01, 15/10/1980, L 1; Aterno, 4709213N, 1861868E, Raiale, 8/9/1995, L 6; Vetoio, 23/3/1992, L 1; Aterno Coppito, 8/5/1992, L 1; Vera, 12/3/1990, L 20; Vera, 1/4/1990, L 150; Vera, 2/4/1990, L 10; Vera, 11/4/1990, L 130; Vera, 12/4/1990, L 30; Vera, 4/5/1990, L 20; Aterno Coppito, 1/5/1989, L 1; Vera, 3/5/1989, L 40; Aterno Coppito, 4/5/1989, L 1; Vera, 6/6/1989, L 80; Vera, 20/7/1989, L 10; Vera, 30/1/1988, L 10; Vera, 12/3/1988, L 20; Vera, 26/3/1988, L 40; Vera, 9/4/1988, L 100; Vera, 23/4/1988, L 10; Vera, 7/5/1988, L 130; Vera, 29/6/1987, L 40; Vera, 18/12/1987, L 20; Nemi, 4623169N, 1807899E, Nemi Nemi, 15/0/1982, L 247; Nemi Nemi, 0/8/1982, L 22; Sangro river, 4661017N, 1897592E, PNA08 Villetta Barrea, 24/1/1990, L 1; Sangro14, 24/1/1990, L 1; PNA02, 19/5/1978, ํ P 1; PNA05, 20/5/1978, L 18; PNA13, 21/5/1978, L 2; Sagittario stream, 4661017N, 1897592E, PNA08 Tasso, 11/11/1988, L 20; PNA08 Tasso spring, 21/5/1978, L 36; Tordino stream, 4734605N, 1885207E, Tord07, 15/0/1991, L 1; Tord03, 5/4/1991, L 2; Tord04, 9/4/1991, L 1; Tord07, 9/4/1991, L 1; Tord03, 15/5/1991, L 1; Tord07, 15/5/1991, L 1; Tord07, 0/8/1991, L 1; Tord05, 5/12/1990, L 1; Tord06, 24/11/1989, L 4; Tord02, 15/2/1979, L 3; Velino river, 4704207N, 1835998E, Velino01, 17/3/1990, ô P 1; Velino Antrodoco, 17/3/1990, L 50; Sangro river, Zittola stream, 4638802N, 1898838E, Zittola, 26/5/1992, L 30; Zittola, 14/1/1991, L 3; Zittola, 14/6/1991, L 1; Zittola, 12/7/1991, L 17; Zittola, 27/11/1991, L 2; Zittola, 7/6/1990, L 1; Dirillo lake, 4125239N, 2010974E, Amerillo stream, 12/6/1984, L 4; Amerillo stream, 5/10/1983, L 1.

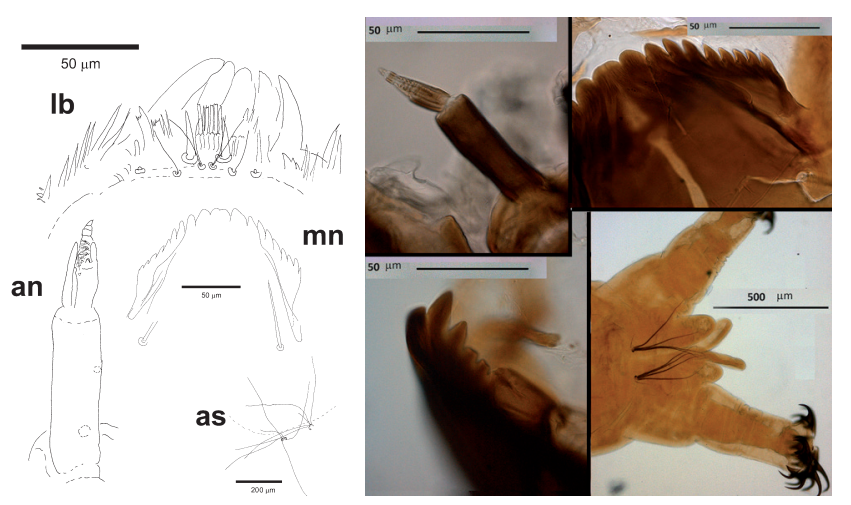

Fig. 13 Left: Diamesa tonsa, antenna, mentum, mandible, caudal end/anal setae; right: Diamesa tonsa, an: antenna - lb: labrum - mn: mentum - as: anal setae.

\section{Key to Diamesa species from Alps and Apennines}

1: Procercus present and developed, with 6 anal setae and 1 lateral seta, setae submenti well caudal with respect to caudal margin of ventromentum, body with very long setae $(>150 \mu \mathrm{m}) \ldots 2$

- Procercus absent or very reduced, with 1-4 anal setae, lateral seta arising directly from the postero-lateral surface on the preanal body segment, setae submenti anterior with respect to caudal margin of ventromental plates or at most $10-15 \mu \mathrm{m}$ posterior ... Diamesa excl dampfi gr 4

2: Head capsule yellow with dark throat and black basal area, 2nd antennal segment bearing 3 well developed sensorial blades (peg sensilla, style and Lauterborn organs (Sæther and Andersen 2013)). Mentum with a group of 3 median teeth similar in size, with well developed lateral expansion of ventromentum, accessory blade shorter than half length of antennal blade, $A R \geq 2.0$ ... Syndiamesa ${ }^{1}$

- Head capsule and throat uniformly yellow, only apex of mentum, mandible and occipital margin black. Mentum with 4 median teeth, often fused in a large tooth, lateral expansion of ventromentum less developed, 2nd antennal segment with 3 sensorial blades of very different length, that is style much longer than the other 2 sensilla (=Lauterborn organs). Larvae with long setae ... D. dampfi gr 3

3: Mentum with 9 lateral teeth, 9th tooth very small. AR about 2, living in cold springs, above $1000 \mathrm{~m}$ asl, in Alps ... D. dampfi

- Mentum with 8-9 lateral teeth, the 9th tooth when present very small. AR about 1.7, living in cold spring at lower altitudes, in the Prealps ... D. permacra

4: Anal setae short, less than $150 \mu \mathrm{m}$ long, thickness variable but always less than $5 \mu \mathrm{m}$, posterior parapods very long (500-700 $\mu \mathrm{m})$ with respect to body length and more than $10 \%$ of body length. Head capsule always black, basal scerite of ungula very developed ... 5

- Anal setae longer, more than $200 \mu \mathrm{m}$ long, posterior parapods longwith respect to body length but less than $10 \%$ of body length. Head capsule yellow, brown, black, basal scerite of ungula less developed ... 9

5: Anal setae very short less than $20 \mu \mathrm{m}$, thick ( $\varnothing$ about $3 \mu \mathrm{m}$ ), spine-like. Posterior parapods very long (up to $1500 \mu \mathrm{m}$ ). AR about 1.6. Mandible with apical and first two inner teeth very long. Mentum with a paired median tooth and 10-11 lateral teeth ... D. steinboecki

- Anal setae or short (less than $30 \mu \mathrm{m}$ ) and thin ( $\varnothing$ less than $2 \mu \mathrm{m}$ ) not spiniform, or longer than $30 \mu \mathrm{m}$, thick and spiniform, but always less than 100-150 $\mu \mathrm{m}$, teeth of mandible with different length ...6

6: 4 spiniform anal setae, at least $40-50 \mu \mathrm{m}$ long, thick ( $\varnothing 3-4 \mu \mathrm{m})$, mentum with a straight or concave median margin ... 7

${ }^{1}$ Included in the key because it can be confused with $D$. dampfi group. 
- Anal setae shorter than $50 \mu \mathrm{m}$, often very short $(10-30 \mu \mathrm{m})$, never spiniform, mental teeth with a convex anterior margin, labrum with SIII with two branches each divided into fine branches, SII with a large scale at its basis ... 8

7: Anal setae about $90 \mu \mathrm{m}$ long, thick, $\varnothing 4 \mu \mathrm{m}$, AR high, about 2, labrum with bifid setae SIII, mentum with the median teeth arranged in a straight line, median tooth paired with 12 lateral teeth, setae submenti posterior to caudal margin of ventromentum, body with setae about $70 \mu \mathrm{m}$ long ... D. aberrata

- Anal setae shorter, about $70 \mu \mathrm{m}$ long, thick, $\varnothing 3 \mu \mathrm{m}$, AR lower, about 1.5, labrum with setae SIII divided in more branches, mentum with a concave median margin, a paired median tooth (single when worn out), a 2 nd lateral tooth longer than the 1st and 10-11 lateral teeth (the 11 th is often very reduced), mandible with lateral teeth very long, length of mandibular teeth in the sequence 2nd $>1$ st $>3$ rd $>4$ th $\ldots$ D. bertrami

8: Four anal setae on both sides, $\leq 60 \mu \mathrm{m}$ long, fine $(\varnothing 1 \mu \mathrm{m})$, but longer than $20 \mu \mathrm{m}$, mentum with a paired median tooth and 9 (10) lateral teeth (a single large median tooth when worn out), a convex anterior margin, AR about 1.2 ... D. latitarsis, D. modesta

- Anal setae 4-3 on the right side, 3-3 on the left side, thin and short (at most $20 \mu \mathrm{m}$ long), mandible with 1st lateral tooth more developed than others, mentum with a paired median tooth and 10 lateral teeth, AR about 1.2 ... D. goetghebueri

9: Setae anteriores SIII simple, setae submenti posterior to caudal margin of ventromentum. Head capsule yellow with a large black occipital margin, mandible and distal part of mentum black ... D. starmachi

- Setae anteriores SIII bifid, setae submenti never caudal with respect to ventromentum, head with less extended black occipital margin ... 10

10: Antenna with the 3rd segment apparently not annulated, but sclerotization present as 2 large rings, mentum with the 4th median teeth forming a plate well separated from the other lateral teeth, head capsule with a characteristic light brown color, AR high, $>2$, setae submenti posterior to caudal margin of ventromentum ... D. incallida

- Antenna with annulated 3rd segment ... 11

11: Head capsule entirely black, a light area is observable only around eyes, AR about 1.7. Mentum with a notched median tooth and 9-10 laterals, anal setae shorter, generally less than $200 \mu \mathrm{m}$ long ... D. zernyi, D. vaillanti.

- Head capsule entirely yellow or with extended brown black areas, never completely black, AR $>1.8$. Mentum with a single median tooth often with a notch, with 9-11 laterals, anal setae longer, about $300 \mu \mathrm{m}$ long ... 12

12: Head capsule entirely yellow, except occipital margin, mental teeth and apex of mandible, antennae dark; $\mathrm{AR}<1.70$. Mentum with a single median tooth and 11 laterals, less cold stenothermal, not found in glacial streams ... D. insignipes
- Head capsule yellow or with extended brown areas; AR higher, about 1.8-2.0, mentum with a notched median tooth and 9 laterals ... 13

13: Head capsule entirely yellow except tips of mandibles and mentum, widespread, in glacial streams but also in cold lowland streams ... D. cinerella

- Head capsule with extended brown-black areas ... D. tonsa

\section{Discussion}

At present 107 species worldwide are included in the genus Diamesa (Ashe and Cranston 1990; Ashe and O'Connor 2009). Most species are described in the adult and pupal stage, with a much lower number described in the larval stage. Old papers describing larvae still preserve validity (Potthast 1914; Thienemann and Mayer 1933; Thienemann 1934, 1952; Chernovskii 1949; Wülker 1959; Styczynskj and Rakusa-Suszczewski 1963; Pankratova 1973; Rossaro 1980; Willassen and Cranston 1986), some are in languages other than English (Ferrarese and Rossaro 1981; Makarchenko 1985; Janecek 1998; Lencioni et al. 2007). Doughman (1983) is probably the most comprehensive key to larvae for North American species, while Schmid (1993) and Janecek (1998) are the most recent keys to European species. More recent papers describing larvae are very scanty (Casas and Langton 2001); Sæther and Andersen (2013) gave a key to genera within Diamesinae.

The use of morphometric characters to separate species is generally well accepted by taxonomists and was frequently used in Chironomid studies. However, there is a high variability within species (Willassen 1986). This is particularly true in Diamesa species, in which life cycles higher than one year are possible, with the consequence that specimens belonging to the four larval instars are of very different size, the larger belonging to populations with univoltine or semivoltine life cycles (Willassen 1986). This does not contradict the importance of metric characters, as anal setae length and thickness, antennal ratio, blade, accessory blade and style length, whose validity was confirmed in the present analysis. Conversely the present study emphasizes that the development of mental and mandibular teeth, described as a valuable character in previous works, is difficult to use, because often the teeth are worn excessively in specimens collected in the field.

Qualitative characters as head capsule color (Thienemann 1952), simple, bifid or multi-branched labral setae (Sæther and Andersen 2013) are also important. To sum up, on the basis of larval morphological characters alone, we can conclude: $D$. dampfi and D. permacra are very difficult to be separated to each other, but different ecology may aid (see below); D. steinboecki, D. goetghebueri, $D$. latitarsis, D. bertrami and D. aberrata are similar in the larval stage, but can be separated because of the shape of anal and labral setae, mentum and mandible (the latter two when well preserved); D. cinerella, D. tonsa, D. ze- 
rnyi/D. vaillanti and D. insignipes can be separated from the head capsule color and to a lesser extent by the length of anal setae and A.R. (D. zernyi and D. vaillanti cannot be separated). $D$. incallida and $D$. starmachi larvae are easily distinguishable from all the other species. In any case the collection of pupal exuviae and adult males is always recommended to confirm identifications.

Phylogenetic considerations based on morphological characters require having data on larvae but also on adult males and pupal exuviae that, differently from larvae, have been well described (Serra-Tosio 1973; Kownacki 1980). However, the information provided by adult, pupal exuviae and larval characters often gives contrasting information. Adult males and larvae in particular suggest very different groupings. Restricting the discussion to the presently described species, the most contrasting evidence is probably the one given by the steinboecki group, created on the basis of adult males (Kownacki 1980), with D. steinboecki and $D$. starmachi very similar, but their larvae are quite different. Other contrasting evidence is given by $D$. incallid $a$ and D. aberrata, joined by many similarities as adult males and pupae (Serra-Tosio 1973), but with quite different larvae. The opposite is true for D. steinboecki, D. goetghebueri, $D$. latitarsis, D. bertrami and D. aberrata, with rather similar larvae but very different adults and pupae.

In this context building a phylogenetic tree of the genus Diamesa is a difficult task. When molecular data become available, this information will give some light about the matter, but at present molecular studies considering Diamesa species are very scanty and do not aid so much (Willassen 2005; Cranston et al. 2012).

All the species belonging to the genus Diamesa live in cold springs or streams, however our datasets emphasize autoecological differences among the different species (intra- and inter-groups). This may aid in separating larvae with very similar morphology, so ecological information was added in the keys. For example, the Material examined emphasizes that some species, as $D$. tonsa, are very common and widespread in the Alps and Apennines, while others, as $D$. starmachi, seem to be restricted to very few localities, $D$. incallida, $D$. dampfi and $D$. permacra inhabit cold springs, but $D$. permacra is less cold-stenothermal, D. steinboecki and D. goetghebueri are characteristic of glacial streams. D. insignipes is less cold stenothermal than $D$. cinerella and D. zernyi Serra-Tosio 1973) and is more common in Apennines (Lencioni and Rossaro 2005; Rossaro et al. 2006). The distribution and habitat of species identified as larvae, confirms the information based on the distribution and habitat of species identified as adults and pupal exuviae (Serra-Tosio 1973) with some contrasting evidence. For example, D. steinboecki was not considered characteristic of glacial streams by Serra-Tosio (1973), but of cold springs, while larvae collected in the present research emphasize that the species is really characteristic of glacial streams, as stated by older authors (Thienemann 1954).

\section{Acknowledgements}

The authors wish to thank the Systematics Research Fund, jointly administered by the Linnean Society of the London and the Systematics Association, the Equipment Reserve Funds of Università degli Studi di Milano, and the MUSE-Museo delle Scienze of Trento for financial support. We wish to thank all colleagues who send specimens for comparisons, in particular dr. Brigitte LodsCrozet (Epalinges, Switzerland), dr. Djuradj Milosevic (Niš, Serbia), Jon Olafsson (Reykjavik, Iceland) and the Institut Royal Sciences Naturales Belgique.

\section{REFERENCES}

Ashe P, Cranston PS (1990) Family Chironomidae. In: Soós Á, Papp L (eds) Catalogue of Palaearctic Diptera. Volume 2. Akadémiai Kiadó, Budapest, pp. 113-355.

Ashe P, O'Connor JP (2009) A World Catalogue of Chironomidae (Diptera). Part 1. Buchonomyiinae, Chilenomyiinae, Podonominae, Aphroteniinae, Tanypodinae, Usambaromyiinae, Diamesinae, Prodiamesinae and Telmatogetoninae. Irish Biogeographical Society and National Museum of Ireland, Dublin.

Casas JJ, Langton PH (2001) The larva and pupa of Diamesa veletensis Serra-Tosio, 1971 (Diptera: Chironomidae). Entomol Gaz 52: 117-124.

Chernovskii AA (1949) Opredelitel' lichinok komarov semeistva Tendipedidae. Opredeliteli po faune SSSR, Izdavaemye Zoologicheskim Institutom Akademii Nauk SSSR, 31, Izdatel'stvo Akademii Nauk SSSR, Moscow and Leningrad (St. Petersburg), English translation in Marshall KE, Freshwater Biological Association.

Cranston P, Hardy NB, Morse GE (2012) Molecular Phylogeny for the Chironomidae (Diptera) Syst Entomol 37: 172-188.

Doughman JS (1983) A guide to the larvae of the Nearctic Diamesinae (Diptera: Chironomidae). The genera Boreoheptagyia, Boreoheptagyia, Diamesa, and Pseudokiefferiella. Water Resources Investigations Report, U. S. Geological Survey, 83-4006: IV.

Ferrarese U, Rossaro B (1981) Chironomidi, 1 (Diptera, Chironomidae: Generalità, Diamesinae, Prodiamesinae) 12. In: Ruffo $S$ (ed) Guide Per Il Riconoscimento Delle Specie Animali Delle Acque Interne Italiane, 12: 1-97.

Janecek BFR (1998) Diptera: Chironomidae (Zuckmücken) Larven. Fauna Aquatica Austriaca, Taxonomie und Ökologie aquatischer wirbelloser Organismen (Teil V). Universität für Bodenkultur, Abt. Hydrobiologie (editor), Wien, Austria.

Kownacki A (1980) Ecology and biogeography of the Diamesa steinboecki group. In: Lellák J (ed) Proc. 6th Int. Symp. Chironomidae, Prague, 17-20 August 1976. Acta Univ Carol Biol 1978: 95-102.

Langton PH (1991) A key to pupal exuviae of West Palaearctic Chironomidae. Huntingdon, Cambridgeshire, England, PH Langton.

Langton PH, Visser H (2003) Chironomid exuviae: A key to the pupal exuviae of the West Palaeartic Region. CD-ROM, Expert Center for Taxonomic Identification, Amsterdam.

Lencioni V, Rossaro B (2005) Microdistribution of chironomids (Diptera: Chironomidae) in Alpine streams: an autoecological perspective. Hydrobiologia 533: 61-76.

Lencioni V, Marziali L, Rossaro B (eds) (2007) I Ditteri Chironomidi: morfologia, tassonomia, ecologia, fisiologia e zoogeografia. Quaderni del Museo Tridentino di Scienze Naturali 1. 
Makarchenko EA (1985) Khironomidy Dal'nego Vostoka SSSR. Podsemeistva Podonominae, Diamesinae i Prodiamesinae (Diptera, Chironomidae). (Chironomids of the Far East of the USSR. Subfamilies Podonominae, Diamesinae and Prodiamesinae (Diptera, Chironomidae) Dal'nevostoch nauchn Tsentr Akad Nauk SSSR, Vladivostok, VIII pl.

Pagast $f$ (1947) Systematik und Verbreitung der um die Gattung Diamesa gruppierten Chironomiden Arch. Hydrobiol 41: 435-596.

Pankratova VY (1973) Key to the larvae of the species of the genera Diamesa, Eukiefferiella, Orthocladius, Cricotopus, Psectrocladius and Chaetocladius. (Horne, J. E. J', transl ). B. A. Transl. (N. S.) 64: 1-14.

Potthast A (1914) Über die Metamorphose der Orthocladius-Gruppe. Ein Beitrag zur Kenntnis der Chironomiden Arch. Hydrobiologia Suppl 2: 243-376.

Rossaro B (1980) Description of some unknown larvae of Diamesa genus and corrections to previous descriptions (Diptera, Chironomidae) Arch Hydrobiologia 90: 298-308.

Rossaro B, Lencioni V, Boggero A, Marziali L (2006) Chironomids from southern Alpine running waters: ecology, biogeography. Hydrobiologia 562: 231-246.

Sæther OA, Andersen T (2013) The larvae of Diamesinae (Diptera: Chironomidae) of the Holarctic Region - Keys and diagnoses. In: Andersen T, Cranston PS, Epler JH (eds) Chironomidae of the Holarctic Region. Keys and Diagnoses. Larvae. Insect Syst Evol Suppl 66: 145-178.

Schmid PE (1993) A key to the larval Chironomidae and their instars from Austrian Danube region streams and rivers with particular reference to a numerical taxonomic approach. Part I. Diamesinae, Prodiamesinae and Orthocladiinae. Wass Abwass Suppl 3/93: 1-514.
Serra-Tosio B (1973) Ecologìe et biogéographie des Diamesini d'Europe (Diptera, Chironomidae). Trav Lab Hydrobiol Grenoble 63: 5-175.

Styczynskj B, Rakusa-Suszczewski S (1963) Tendipedidae of selected water habitats, of Hornsund region (Spitzbergen). Polskie Arch Hydrohiol II: 327-341.

Thienemann A (1934) Chironomiden-Metamorphosen. VII. Die Diamesa-Gruppe (Dipt.) Stettin ent Ztg 95: 1-23.

Thienemann A (1952) Bestimmungstabelle für die Larven der mit Diamesa nächst verwandten Chironomiden Beitr Ent 2: 244-256.

Thienemann A (1954) Chironomus. Leben, Verbreitung und wirtschaftliche Bedeutung der Chironomiden Binnengewässer 20.

Thienemann A, Mayer K (1933) Chironomiden-Metamorphosen. VI. Die Metamorphosen zweier hochalpiner Chironomiden (Dipt.). (Mit einer Bestimmungstabelle der Larven und Puppen der Diamesa-Gruppe) Zool Anz 103: 1-12.

Willassen E (1985) A review of Diamesa davisi Edwards and the davisi group (Diptera, Chironomidae). In: Fittkau EJ (ed) Beiträge zur Systematik der Chironomidae, Diptera. Spixiana Suppl 11: 109-137.

Willassen E (2005) New species of Diamesa (Diptera: Chironomidae) from Tibet: conspecific males and females associated with mitochondrial DNA. Zootaxa 1049: 19-32.

Willassen E, Cranston PS (1986) Afrotropical montane midges (Diptera, Chironomidae, Diamesa) Zool J Linn Soc 87: 91-123.

Wirth WW, Marston N (1968) A method for mounting small insects on microscope slides in Canada Balsam. Ann Entomol Soc Am 61: 783-784.

Wülker W (1959) Diamesarien-Studien (Dipt., Chironomidae) im Hochschwarzwald. Arch Hydrobiol Suppl 24: 338-360. 